\title{
Credit risk analysis with creditor's option to extend maturities
}

\author{
Ryoichi Ikeda $^{1} \cdot$ Yoske Igarashi $^{2}$ (D)
}

Received: 27 June 2016 / Accepted: 13 September 2016 / Published online: 8 October 2016 (C) The Author(s) 2016. This article is published with open access at Springerlink.com

\begin{abstract}
We present a Merton (J Finance, 1974)-type structural model of credit risk in which the borrower firm refinances its debt, there is cost for bankruptcy, and the creditor has an option to extend the date of maturity of debt if the firm defaults. We show that a solution exists in such a model and in that solution the creditor has incentive to extend maturity to avoid bankruptcy cost. We solve the model numerically and argue that such maturity extension option for the creditor can have substantial impact on the debt and stock values of the firm.
\end{abstract}

Keywords Structural model · Default · Bankruptcy cost · Maturity extension · Refinancing

JEL Classification G12 $\cdot \mathrm{G} 13 \cdot \mathrm{G} 21 \cdot \mathrm{G} 33$

\section{Introduction}

This article studies corporate risk when the substantial part of a firm's debt is financed by one (or few) major creditor(s) along with other minute creditors. In the literature

We are extremely grateful to the referee who made helpful comments and advice. This research is supported by JSPS KAKENHI Grant Number JP 1617150 and by the FMV cluster funding of the University of Exeter Business School for the 2015-16 academic year.

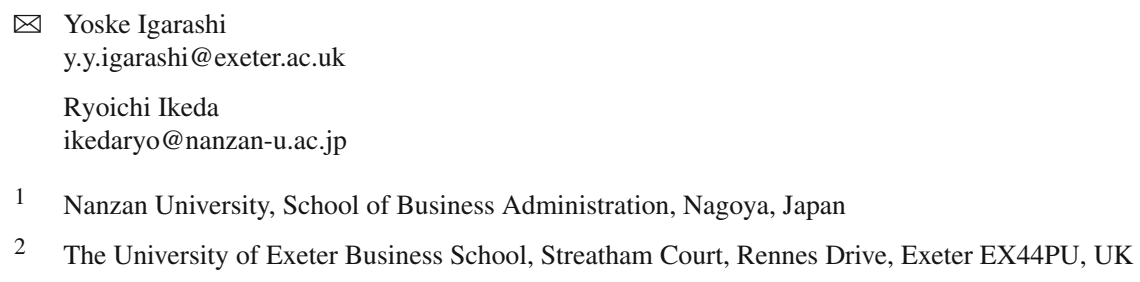


of structural models of corporate risk that follows Merton (1974) and Black and Cox (1976), a single creditor is typically assumed for simplification. In reality, if there is only one creditor, the creditor has an option of flexible private renegotiation whenever the firm encounters financial distress. For instance, under Japan's so-called 'main bank system', the main bank of a firm often chooses private renegotiation rather than legal renegotiation that follows application of the Japan's Corporate Reorganization Law. ${ }^{1}$ This can have several implications on the firm's debt and stock values and its default and bankruptcy probabilities.

We present a Merton (1974)-type structural model of credit risk in which the firm (debtor) refinances its debt, there is cost for bankruptcy, and the creditor has an option to extend maturity, one of the simplest forms of private renegotiation. ${ }^{2}$ In our model, the firm's debt is in the form of a short-term discount bond with a fixed face value and fixed time to maturity. Every time the maturity of the debt contract arrives, the firm (i.e., stockholders) chooses whether to repay through refinancing (issuing a new debt and putting up more equity capital) or simply to default on the debt. If default is chosen by the firm, then the creditor chooses whether to force bankruptcy or not. That is, if extending the maturity of the debt repayment and making the firm continue as a going concern leads to debt value that is higher than the payoff of bankruptcy (the firm's asset value minus cost of bankruptcy), the creditor chooses to do so. In other words, the model features a two-sided option, the firm's decision for default and the creditor's decision for bankruptcy. The latter implies that default does not necessarily lead to bankruptcy, so default and bankruptcy become two distinct events.

By making some restrictions on what the creditor can do in the event of the firm's default, we can prove the existence of a solution to the model that possesses certain characteristics. Then we numerically solve the model and compute the debt and stock values of the firm and its default and bankruptcy probabilities. We find that when the firm defaults on debt, the creditor might extend the maturity of debt if the firm's asset is so low that the creditor cannot get much from liquidation.

Effects of such maturity extension include the following. First, when the bankruptcy cost is high, the presence of such a maturity extension option can lead to higher debt value than in the absence of such an option. Second, because of the higher possibility of avoiding bankruptcy, stock value can also be higher. Third, the bankruptcy probability can be substantially lower. This is important for other minute creditors of the firm; without taking into account the main creditor's maturity extension option, they would overestimate the firm's bankruptcy probability. Finally, the default probability can be lower with the presence of possible maturity extension. This is because the reduction of the bankruptcy probability raises the stock value, which in turn makes refinancing easier. That is, the effect of the bank's maturity extension option on the firm's bankruptcy risk is not only through the obvious channel that extension simply could prevent immediate bankruptcy but also through a kind of 'multiplier effect'

\footnotetext{
1 Detailed studies of the roles and incentives of the main bank in the event of the debtor's financial distress are found in Aoki and Hugh (1995).

2 Refinancing is modeled in Leland and Toft (1996) but in order to explicitly model the creditor's option to postpone the firm's repayment after default, we rather build on Merton (1974).
} 
that the reduction of long-run bankruptcy probability raises the stock value, facilitates refinancing, lowers default probability, and further pushes down bankruptcy risk.

As a feature of models with refinancing, the effect of asset volatility (i.e., risk-taking of a firm) also differs from that in the standard Merton model; that is, the increase in volatility has a non-monotonic effect on the firm's debt and stock values. Specifically, stockholders avert risk when the asset level is high, and the creditor likes risk when the asset level is relatively low. The former is because high volatility lowers the debt value and worsens refinancing condition for stockholders. The latter is because when the asset level is relatively low, high volatility raises the stock price, which makes the firm's refinancing easier and the default probability lower. Adding the creditor's maturity extension option to the model magnifies this non-monotonicity.

In some models in the literature, the trigger of default, or the threshold of the borrower firm's asset level below which the firm defaults, is exogenously given. ${ }^{3}$ Other models such as Leland (1994) and Leland and Toft (1996) have a one-sided option; stockholders choose such a threshold but the creditor does not have any option. Stockholders in these models make decisions whether to repay the debt to maintain the firm or whether to go bankrupt and transfer the ownership to the creditor. ${ }^{4}$ When the bankruptcy cost is high, however, there can be situations that the stockholders give up repayment and choose bankruptcy but the creditor wants to maintain the firm even by offering postponement of repayment, and this yields models with a two-sided option. However, there are not many pricing models of corporate bonds in which the creditor can renegotiate over the repayment condition of the debtor. Among the few such pricing models are Mella-Barral and Perraudin (1997) and Fan and Sundaresan (2000). These models consider a firm that issues 'consols' and do not consider refinancing. In our model, the firm refinances a discount bond by issuing a new bond, the value of which is low when the asset level of the firm is low. One can interpret the firm of our model as issuing 'consols' with variable coupons whose amount increases as the credit risk of the firm increases.

The structure of the rest of the paper is as follows. In Sect. 2, we present our model in several versions and establish existence of solutions. Section 3 numerically computes solutions and gives discussion. Section 4 concludes.

\section{Model}

\subsection{Common environment}

There is a firm whose date- $t$ asset value $A_{t}$ follows a geometric Brownian motion under a risk-neutral probability measure:

$$
d A_{t}=A_{t}\left(r d t+\sigma d W_{t}\right)
$$

\footnotetext{
3 See for example Black and Cox (1976), Kim et al. (1993) and Longstaff and Schwartz (1995).

4 With the presence of bankruptcy cost and tax benefit of debt, the Modigliani-Miller theorem does not hold and the optimal capital structure becomes an issue, which is the main interest of Leland (1994) and Leland and Toft (1996). In this paper, we abstract from the optimal capital structure problem.
} 
where $\sigma$ is the volatility, $r$ is the risk-free rate, and $W_{t}$ is a standard Brownian motion with respect to the risk-neutral probability. The firm owes a single creditor (e.g. "main bank") a debt with face value $f$ and time to maturity $\Delta$ years. To abstract from the optimal capital structure choice problem, we assume that both $f$ and $\Delta$ are exogenously given to the firm for some institutional reasons. Once the maturity arrives, the firm repays the debt by the following refinancing process. First, the firm rolls over the debt; that is, the firm makes another debt contract again with face value $f$ and time to maturity $\Delta$. Because the amount of funds raised from this new debt contract is not enough to repay $f$, the firm fills the gap by raising equity capital. ${ }^{5}$ As long as the firm stays solvent, this refinancing process is repeated every $\Delta$ years. ${ }^{6}$ Since the face value of the firm's debt always remains constant at $f$, the model has a kind of time-homogeneous structure; nothing depends on the calendar time but only on the remaining time to the maturity of debt.

If the firm's asset value, say $\tilde{A}$, at the date of debt maturity is very low, the firm cannot repay the debt; that is, the amount of funds the firm can raise from re-borrowing and stock issuance does not reach $f{ }^{7}$ We call that situation default. In the event of default, the creditor has two options: bankruptcy and postponement. If the creditor chooses the bankruptcy option, it lets the firm go bankrupt and receives $\min (f, \alpha \tilde{A})$, where $\alpha \in(0,1]$ is the asset recovery rate. The idea is that bankruptcy is costly; if the firm's asset level upon default is $\tilde{A}$, then $1-\alpha$ proportion is lost as bankruptcy cost and only $\alpha$ proportion is received by the creditor (and potentially by stockholders if $\alpha \tilde{A}>f$ ). On the other hand, if the creditor chooses the postponement option to extend the date of debt maturity, it keeps the same debt but the new date of maturity comes in another $\Delta$ years.

In Sect. 2.2, we will consider four versions of the model with refinancing that differ in assumptions on the creditor's postponement option. In Model I, the creditor does not have the option to extend the maturity date and thus always forces bankruptcy after default. Hence this version resembles Leland and Toft (1996) while it still differs from their model in that the date of maturity only arrives every $\Delta$ years while it arrives continuously in their model. In Models II and III, the creditor has the option to extend the maturity date but only $N$ times, where $N$ is an exogenously given bound. The difference between II and III is whether or not the remaining number of postponements allowed is reset to $N$ once the firm successfully repays the debt. In Model IV, the creditor can extend the maturity date an unlimited number of times.

One of our goals is to demonstrate possible impacts of maturity extension in a simple model and provide complete proof for existence of a solution that applies a fixed-point

\footnotetext{
5 The firm and the stockholder can be identical (e.g. "owner-manager"), in which case issuing new stocks means additional injection of money out of his pocket.

6 The assumption that the debt is repaid by issuing new stocks is found, for instance, in Leland (1994) and Brennan and Schwartz (1978) and is necessary for the firm's asset $A$ to be exogenous (unaffected by the capital structure of the firm).

7 In the case of the 'owner-manager', this means that he does not find it in his interest to meet the debt obligation by putting up additional equity capital out of his own pocket.
} 
theorem. ${ }^{8}$ For that purpose, we have made several restrictive assumptions as above on what the creditor can do. First, our model has no benefit of debt and hence does not allow to study the optimal amount of debt (or optimal capital structure), so a fixed amount of debt is imposed. While this simplification seems to be a good step toward a model in which the amount of debt is endogenous, it remains an open question whether such a full-fledged model has very different implications. Second, it is assumed that the length of extension is always $\Delta$. In reality, the creditor should choose between, for example, extending by 1 month and extending by 1 year. However, we can assume in our framework that $\Delta$ is short (such as 1 month) and $N$ is, say, twelve. In such an environment, the creditor is allowed to extend the maturity both for 1 month and for 12 months in total, which resembles the maturity choice by the creditor. Third, $N$, the maximum number of maturity extensions the creditor can give, is exogenously fixed in Models II and III. Although it is certainly less restrictive than existing models that do not allow maturity extension at all, it may appear somewhat arbitrary. One interpretation is that some past history or the creditor's reputation has led investors to expect that the creditor gives extension at most a fixed number of times and not unlimitedly. We consider the case of an unlimited number of maturity extensions in Model IV.

\subsection{Model variations and propositions}

We focus on the prices of debt and stock right after the refinancing/maturity extension: that is, when the time to debt maturity is exactly $\Delta$ years. ${ }^{9}$ Let $A$ be the level of the firm asset today and $\tilde{A}$ be the (future) asset value at the date of debt maturity, which is $\Delta$ years from today. Throughout the section, $E_{\tilde{A} \mid A}[\cdot]$ denotes expectation with respect to the risk-neutral probability conditional on the current asset level $A$. All proofs of propositions are in the "Appendix".

\section{Model I: no postponement}

We start with the case where the creditor does not have the option to extend the maturity on the firm's default, that is, when $N=0$. In this case, the firm's default always leads to bankruptcy. Let $F^{0}(A)$ and $S^{0}(A)$ denote the debt and stock values when the current level of the firm asset is $A$ and the time to maturity of the debt is $\Delta$ years. When the maturity arrives and the firm asset value at that time is $\tilde{A}$, the firm repays $f$ by issuing a new debt valued $F^{0}(\tilde{A})$, and new stocks that are worth $f-F^{0}(\tilde{A})$. After this process, the total stock value is $S^{0}(\tilde{A})$ and hence the value of old stocks is the difference $S^{0}(\tilde{A})-\left(f-F^{0}(\tilde{A})\right)$. This means that if this difference is nonnegative, the

\footnotetext{
8 To the best of our knowledge, our proof approach for existence of a solution in credit risk models that have some recursive structure is new in the literature.

9 Prices at different times are obtained by discounting by using the risk neutral probability for the remaining time to maturity.
} 
firm has positive net worth and can be successfully refinanced (hence no default). ${ }^{10}$ Otherwise, the firm is bankrupt and the creditor recovers $\min (f, \alpha \tilde{A})$. Let $\bar{A}^{0}$ denote the default threshold, defined by

$$
S^{0}(\tilde{A})-\left(f-F^{0}(\tilde{A})\right)\left\{\begin{array}{ll}
>0 & \text { if } \tilde{A}>\bar{A}^{0} \\
=0 & \text { if } \tilde{A}=\bar{A}^{0} \\
<0 & \text { if } \tilde{A}<\bar{A}^{0}
\end{array} .\right.
$$

Then, as we explain below, the default threshold and the security prices should satisfy the following system of integral equations:

$$
\begin{aligned}
& F^{0}(A)=e^{-r \Delta} E_{\tilde{A} \mid A}\left[\mathbf{1}_{\left\{\tilde{A} \geq \bar{A}^{0}\right\}} f+\mathbf{1}_{\left\{\tilde{A}<\bar{A}^{0}\right\}} \alpha \tilde{A}\right], \quad \forall A>0, \\
& S^{0}(A)=e^{-r \Delta} E_{\tilde{A} \mid A}\left[\mathbf{1}_{\left\{\tilde{A} \geq \bar{A}^{0}\right\}}\left(S^{0}(\tilde{A})-\left(f-F^{0}(\tilde{A})\right)\right)\right], \quad \forall A>0, \\
& F^{0}\left(\bar{A}^{0}\right)+S^{0}\left(\bar{A}^{0}\right)=f .
\end{aligned}
$$

where $\mathbf{1}_{\{\ldots\}}$ is the indicator function.

The interpretation of (3)-(5) is as follows. Any security price is the discounted future payoffs, discounted by the risk-free rate and the risk-neutral probability. The right side of (3) means that the future payoff to the creditor is $f$ if the future asset level $\tilde{A}$ is above the default threshold $\bar{A}^{0}$, and it is $\alpha \tilde{A}$ otherwise. The right side of (4) means that the future payoff to the stockholders is $S^{0}(\tilde{A})-\left(f-F^{0}(\tilde{A})\right)$ if the future asset level is above the default threshold and is zero otherwise. Equation (5) only re-states the equality in (2). ${ }^{11}$ We have the following proposition.

Proposition 2.1 A solution $\left(F^{0}(\cdot), S^{0}(\cdot), \bar{A}^{0}\right)$ to (3)-(5) exists, and it satisfies

(i) $\alpha A<F^{0}(A)+S^{0}(A)<A, \quad \forall A>0$,

(ii) $\alpha \bar{A}^{0}<f<\bar{A}^{0}$, and

(iii) $F^{0}(\cdot)+S^{0}(\cdot)$ is strictly increasing.

The inequalities (i) are somewhat intuitive because no matter when cash flow occurs, the sum of payments to the creditor and stockholders never exceeds $\tilde{A}$ or goes below $\alpha \tilde{A}$, whose present values by the risk-neutral probability measure are $A$ and $\alpha A$, respectively. The inequality (ii) follows (i) and (5) and implies $\min (f, \alpha \tilde{A})=\alpha \tilde{A}$ if $\tilde{A}<\bar{A}^{0}$. Finally, (iii) confirms that the default threshold is well-defined by (2). Note that with the presence of bankruptcy cost, there is a region for $\tilde{A}$ that is higher than the debt face value but still leads to default and bankruptcy, namely $\left[f, \bar{A}^{0}\right.$ ).

Due to the existence of bankruptcy cost and the absence of positive sides of debt such as tax advantage, the firm's value defined as the sum of the stock and debt values

\footnotetext{
10 In this model, new stock issuance is not for brand-new investment opportunities, but to simply replace the debt that finances existing projects. For this reason, the total value of outstanding stocks after successful refinancing is simply the value of the original stocks plus the money paid by the new stockholders.

11 Eqs. (3)-(4) assume $\min (f, \alpha \tilde{A})=\alpha \tilde{A}$, whenever bankruptcy occurs. This is confirmed to be the case both in our propositions and in the numerical solution in Sect. 3.
} 
is strictly smaller than the firm's asset value $A$ [see (i)]. Consequently, the optimal capital structure in the model would be 'no debt at all' if $f$ were not exogenous to the firm. ${ }^{12}$

Equation (3) implies that once the threshold $\bar{A}^{0}$ is given, we can obtain the analytical solution for the debt value: ${ }^{13}$

$$
F^{0}\left(A ; \bar{A}^{0}\right)=\alpha A \Phi\left(-x_{1}\right)+f e^{-r \Delta} \Phi\left(x_{2}\right),
$$

where $\Phi(\cdot)$ is the cumulative density function of the standard normal distribution and

$$
x_{1}, x_{2}=\frac{\log \left(A / \bar{A}^{0}\right)+\left(r \pm \sigma^{2} / 2\right) \Delta}{\sigma \sqrt{\Delta}} .
$$

In the special case of $\alpha=1$, the solution satisfies $F^{0}(A)+S^{0}(A)=A, \forall A>0$ and (ii) reduces to $\bar{A}^{0}=f$. In this case, (6)-(7) coincide with Merton (1974), a model without refinancing. If $\alpha<1$, the present model differs from the Merton model even if the latter is augmented with the same bankruptcy cost. This is because in our model of refinancing the default threshold is strictly greater than $f$, while it remains $f$ in the Merton model.

We conclude Model I with the following proposition.

Proposition 2.2 There exists a unique $\underline{A}^{1}>0$ such that $\tilde{A} \lesseqgtr \underline{A}^{1} \Leftrightarrow F^{0}(\tilde{A}) \gtreqless$ $\alpha \tilde{A}, \forall \tilde{A}$.

As will become clear, this proposition indicates that if the creditor were allowed to postpone the firm's repayment once, the creditor would do so if and only if the firm's asset at the point of that decision is below some threshold. We will use the existence of this threshold in models with maturity extension.

\section{Model II: finite postponements without resetting}

In this version of the model, the creditor has an option to extend the maturity date of the debt if the firm defaults. When the firm does not repay the debt at the maturity date, the creditor compares the debt value after such maturity extension is given and the immediate payoff of forcing bankruptcy. If the former is higher, the creditor extends the debt maturity by another $\Delta$ years. After $\Delta$ years, the same situation arises and this process can be repeated until the creditor extends the firm's repayment $N$ times in total, where $N$ is an exogenous bound on the number of times the creditor can postpone the firm's repayment.

Let $F^{N}(A)$ and $S^{N}(A)$ be the debt and stock values when the current level of the firm asset is $A$, the time to maturity is $\Delta$ years, and the number of remaining postponement

\footnotetext{
12 If the firm (stockholder) were allowed not to roll over the debt, when the first maturity arrives, the stockholder would pay off the debt once and for all by paying out of his pocket, if and only if $A \geq f$. After that, the firm will continue as a going concern with no debt. Such a model can be regarded as the Merton model augmented with bankruptcy cost.

13 The derivation is analogous to that of Black-Scholes formula for European put option.
} 
options is $N$. If the firm defaults with asset level $\tilde{A}$ and if $N \geq 1$, the creditor receives $\min (f, \alpha \tilde{A})$ by forcing bankruptcy or $F^{N-1}(\tilde{A})$ by extending the maturity. So the payoff to the creditor is whichever is larger. The default threshold, denoted $\bar{A}^{N}$, is defined in a similar manner to (2) of Model I, namely by $S^{N}\left(\bar{A}^{N}\right)-\left(f-F^{N}\left(\bar{A}^{N}\right)\right)=0$. When $N=0$, this model reduces to Model I. Given $F^{0}(\cdot), S^{0}(\cdot)$, and $\bar{A}^{0}$ defined in Model I, we inductively define the system of integral equations for $N \geq 1$ :

$$
\begin{gathered}
F^{N}(A)=e^{-r \Delta} E_{\tilde{A} \mid A}\left[\mathbf{1}_{\left\{\tilde{A} \geq \bar{A}^{N}\right\}} f+\mathbf{1}_{\left\{\tilde{A}<\bar{A}^{N}\right\}} \max \left(\alpha \tilde{A}, F^{N-1}(\tilde{A})\right)\right], \quad \forall A>0, \\
S^{N}(A)=e^{-r \Delta} E_{\tilde{A} \mid A}\left[\mathbf{1}_{\left\{\tilde{A} \geq \bar{A}^{N}\right\}}\left(S^{N}(\tilde{A})-\left(f-F^{N}(\tilde{A})\right)\right)\right. \\
\left.\left.+\mathbf{1}_{\left\{\tilde{A}<\bar{A}^{N}\right\}} \mathbf{1}_{\left\{\alpha \tilde{A}<F^{N-1}(\tilde{A})\right\}} S^{N-1}(\tilde{A})\right)\right], \quad \forall A>0 \\
F^{N}\left(\bar{A}^{N}\right)+S^{N}\left(\bar{A}^{N}\right)=f .
\end{gathered}
$$

Being analogous to (3)-(4) of Model I, Eq. (8) and (9) imply that both the debt and stock values are the discounted expected future payoffs. Equation (10) is the re-statement of the definition of the default threshold.

Given $\underline{A}^{1}$ obtained in Proposition 2.2, we can show the following proposition.

Proposition 2.3 Suppose that parameters are such that $\bar{A}^{0} \leq \underline{A}^{1}$ holds. Then for each $N$, a solution $\left(F^{N}(\cdot), S^{N}(\cdot), \bar{A}^{N}\right)$ to $(8)-(10)$ exists, and it satisfies:

(i) $\alpha A<F^{N}(A)+S^{N}(A)<A, \forall A>0$,

(ii) $\alpha \bar{A}^{N}<f<\bar{A}^{N}$,

(iii) $F^{N}(\cdot)+S^{N}(\cdot)$ is strictly increasing, and

(iv) the creditor always chooses to exercise the maturity extension option whenever possible.

Moreover, in this solution, the higher $N$ is, the less likely is the next default;

$$
\bar{A}^{0}>\bar{A}^{1}>\cdots>\bar{A}^{N}
$$

and both the debt and stock values are increasing in $N$; that is, for all $A>0$,

$$
\begin{aligned}
& F^{0}(A)<F^{1}(A)<\cdots<F^{N}(A), \text { and } \\
& S^{0}(A)<S^{1}(A)<\cdots<S^{N}(A) .
\end{aligned}
$$

Proof is done inductively, using the result of Model I as the terminal condition.

Consider the $N=1$ case and suppose that the firm defaults for the first time with asset value $\tilde{A}$. The creditor compares the liquidation value $\alpha \tilde{A}$ with the new debt value that it can get by postponing the firm's repayment once and for all, namely $F^{0}(\tilde{A})$. Proposition 2.2 implies that the creditor chooses to postpone if and only if the asset value is lower than $\underline{A}^{1}$, the level we call the postponement threshold. Notice that how likely it is that the firm will be able to repay does not matter much to this decision 
of the creditor. It is when the firm's asset level is sufficiently low that the creditor puts more value on a small chance of the firm recovering from distress than on the immediate cash from liquidating the firm.

We denote the postponement threshold in general as $\underline{A}^{N}, N \in \mathbb{N}$. We have not been able to show analytically the existence of such threshold for general $N$. However, we carried out computation with various parameter values and always found a unique threshold. If such a threshold exists, the postponement-triggering condition in (9), namely $\alpha \tilde{A}<F^{N-1}(\tilde{A})$, is written as $\tilde{A}<\underline{A}^{N}$, and the integral equations are rewritten accordingly. That is, (9) becomes

$$
\begin{aligned}
S^{N}(A)= & e^{-r \Delta} E_{\tilde{A} \mid A}\left[\mathbf{1}_{\left\{\tilde{A} \geq \bar{A}^{N}\right\}}\left(S^{N}(\tilde{A})-\left(f-F^{N}(\tilde{A})\right)\right)\right. \\
& \left.\left.+\mathbf{1}_{\left\{\tilde{A}<\bar{A}^{N}\right\}} \mathbf{1}_{\left\{\tilde{A}<\underline{A}^{N}\right\}} S^{N-1}(\tilde{A})\right)\right], \quad \forall A>0 .
\end{aligned}
$$

\section{Model III: finite postponements with resetting}

In this version of the model, $N$ is an exogenous bound on the number of maturity extensions that the creditor can make consecutively. As was the case with Model II, if the firm defaults and the creditor extends the date of the debt maturity $N$ times in a row, then at the next date of maturity, the creditor can no longer extend the maturity. However, in this version, it is assumed that once the firm repays its debt, the remaining number of possible maturity extensions is reset to $N$ again.

Let $M(0 \leq M \leq N)$ be the remaining number of successive extensions allowed. Let $F^{N, M}(A)$ and $S^{N, M}(A)$ denote the debt and stock values when the current asset level of the firm is $A$ and the time to maturity is $\Delta$ years. Denote the default threshold by $\bar{A}^{R, N}$, where $R$ stands for 'reset'. The threshold is defined, as in (2), by $S^{N, N}\left(\bar{A}^{R, N}\right)-$ $\left(f-F^{N, N}\left(\bar{A}^{R, N}\right)\right)=0$. As is understood in (15) below, the default threshold does not depend on $M$ because once the firm repays, $M$ is reset to $N$, and hence only $S^{N, N}$ and $F^{N, N}$ matter to refinancing. In the event of default, the creditor exercises its maturity extension option if and only if $F^{N, M-1}(\tilde{A})>\alpha \tilde{A}$. The default threshold and the security prices should satisfy the following system of integral equations: $\forall A>0$,

$$
\begin{gathered}
F^{N, M}(A)=\left\{\begin{array}{ll}
e^{-r \Delta} E_{\tilde{A} \mid A}\left[\mathbf{1}_{\{\tilde{A} \geq \bar{A}}, \bar{A}^{R, N}\right\} & \\
e^{-r \Delta} E_{\tilde{A} \mid A}\left[\mathbf{1}_{\left\{\tilde{A} \geq \bar{A}^{R, N}\right\}} f+\mathbf{1}_{\left\{\tilde{A}<\bar{A}^{R, N}\right\}} \alpha \tilde{A}\right] & \text { if } M=0 \\
S^{N, M}(A)=\left\{\begin{array}{c}
e^{-r \Delta} E_{\tilde{A} \mid A}\left[\mathbf{1}_{\left\{\tilde{A} \geq \bar{A}^{R, N}\right\}}\left(S^{N, N}(\tilde{A})-\left(f-F^{N, N}(\tilde{A})\right)\right)\right] \\
e^{-r \Delta} E_{\tilde{A} \mid A}\left[\mathbf{1}_{\left\{\tilde{A} \geq \bar{A}^{R, N}\right\}}\left(S^{N, N}(\tilde{A})-\left(f-F^{N, N}(\tilde{A})\right)\right)\right. \\
\left.\left.+\mathbf{1}_{\left\{\tilde{A}<\bar{A}^{R, N}\right\}} \mathbf{1}_{\left\{\alpha \tilde{A}<F^{N, M-1}(\tilde{A})\right\}} S^{N, M-1}(\tilde{A})\right)\right] \\
F^{N, N}\left(\bar{A}^{R, N}\right)+S^{N, N}\left(\bar{A}^{R, N}\right) 7=f .
\end{array},\right. & \text { if } M \geq 1
\end{array},\right.
\end{gathered}
$$

Proposition 2.4 Suppose that parameters are such that $\bar{A}^{0} \leq \underline{A}^{1}$. Then for each $N$, there exist $\left\{F^{N, M}(\cdot), S^{N, M}(\cdot)\right\}_{M=0,1, \ldots, N}$ and $\bar{A}^{R, N}$ that are a solution to (14)-(16), and such that 
(i) $\alpha A<F^{N, M}(A)+S^{N, M}(A)<A, \quad \forall A>0, \forall M=0,1, \ldots, N$,

(ii) $\alpha \bar{A}^{R, N}<f<\bar{A}^{R, N}<\bar{A}^{0}$,

(iii) $F^{N, M}(\cdot)+S^{N, M}(\cdot)$ is strictly increasing, and

(iv) the creditor always chooses to exercise the maturity extension option whenever possible.

Moreover, both the debt and stock values are increasing in $M$ : that is, for all $A>0$,

$$
\begin{aligned}
& F^{N, 0}(A)<F^{N, 1}(A)<\cdots<F^{N, N}(A), \text { and } \\
& S^{N, 0}(A)<S^{N, 1}(A)<\cdots<S^{N, N}(A) .
\end{aligned}
$$

Although the change from Model II to Model III is seemingly minor, the proof approach is very different from that of Proposition 2.3. This is because for given $N$, the $2(1+N)$ functions $\left\{F^{N, M}(\cdot), S^{N, M}(\cdot)\right\}_{M=0,1, \ldots, N}$ are dependent on one another, so their existence must be established simultaneously, not inductively. Consequently, while properties (i)-(iv) of Proposition 2.3 are carried over to Model III, the monotonicity in terms of $N$, namely (11)-(13), is not. Inequalities (17)-(18) represent monotonicity in terms of the remaining number of postponements, $M$, for a fixed $N$.

\section{Model IV: unlimited postponements}

Lastly, assume that the creditor's option to postpone the firm's repayment in the event of default is unlimited. This corresponds to $N=\infty$ in Models II and III. Therefore, by the analogous logic, the default threshold, denoted $\bar{A}^{\infty}$, and security prices, denoted $F^{\infty}(\cdot)$ and $S^{\infty}(\cdot)$, satisfy the following system of integral equations:

$$
\begin{gathered}
F^{\infty}(A)=e^{-r \Delta} E_{\tilde{A} \mid A}\left[\mathbf{1}_{\left\{\tilde{A} \geq \bar{A}^{\infty}\right\}} f+\mathbf{1}_{\{\tilde{A}<\bar{A}} \max \left(\alpha \tilde{A}, F^{\infty}(\tilde{A})\right)\right], \quad \forall A>0 \\
S^{\infty}(A)=e^{-r \Delta} E_{\tilde{A} \mid A}\left[\mathbf{1}_{\left\{\tilde{A} \geq \bar{A}^{\infty}\right\}}\left(S^{\infty}(\tilde{A})-\left(f-F^{\infty}(\tilde{A})\right)\right)\right. \\
\left.+\mathbf{1}_{\left\{\tilde{A}<\bar{A} \bar{A}^{\infty}\right\}} \mathbf{1}_{\left\{\alpha \tilde{A}<F^{\infty}(\tilde{A})\right\}} S^{\infty}(\tilde{A})\right], \quad \forall A>0 \\
S^{\infty}\left(\bar{A}^{\infty}\right)-\left(f-F^{\infty}\left(\bar{A}^{\infty}\right)\right)=0 .
\end{gathered}
$$

Suppose that the assumption of Propositions 2.3 and 2.4 holds. Proposition 2.3 then implies that as $N$ goes to infinity, $\bar{A}^{N}$ converges to some limit $\bar{A}^{\infty} \geq f$. Also, Proposition 2.3 [(i), (12) and (13)] implies that the debt and stock values also converge to some $F^{\infty}(\cdot)$ and $S^{\infty}(\cdot)$. What happens in the limit is shown by the following proposition and it is somewhat intuitive.

Proposition 2.5 Suppose $\bar{A}^{0}<\underline{A}^{1}$. Then a solution $\left(F^{\infty}(\cdot), S^{\infty}(\cdot), \bar{A}^{\infty}\right)$ to (19)(21) exists, and it satisfies $\bar{A}^{\infty}=\bar{f}$, and $F^{\infty}(A)+S^{\infty}(A)=$ A for all A. Moreover, the creditor always chooses postponement whenever default occurs.

That is, if parameters imply $\bar{A}^{0}<\underline{A}^{1}$, which typically is the case when $\alpha$ is small, the creditor always postpones the firm's repayment following its default and hence 
Table 1 Threshold values of the firm asset

\begin{tabular}{|c|c|c|c|c|}
\hline & Model I & Model II $(N=1)$ & Model III $(N=1)$ & Model IV \\
\hline \multicolumn{5}{|l|}{ (i) $\alpha=0.8$} \\
\hline Default threshold & 1.194 & 1.194 & 1.194 & 1.194 \\
\hline Postponement threshold & - & 0.528 & 0.528 & 0.552 \\
\hline \multicolumn{5}{|l|}{ (ii) $\alpha=0.5$} \\
\hline Default threshold & 1.678 & 1.596 & 1.540 & 1.000 \\
\hline Postponement threshold & - & 1.735 & 1.889 & 1.980 \\
\hline
\end{tabular}

bankruptcy never happens. As Proposition 2.5 shows, in this case the default threshold coincides with the face value of the debt $f$ and the sum of the debt and stock values is equal to the asset value itself, which is the case with the Merton model. However, Model IV still differs from the Merton model even if the latter is augmented by the same bankruptcy cost. When the asset value is below $f$, the payoff to the stockholder is zero in the Merton model but it is not in our model because the postponement option is exercised and the firm continues.

The proposition predicts that for some $\alpha$ (e.g. small $\alpha$ due to very firm-specific asset), default never leads to bankruptcy, so the size of $\alpha$ does not affect the default threshold nor the security values. In reality, the presence of asymmetric information between the firm and the creditor is likely to make the creditor force bankruptcy after the firm defaults a finite number of times. Nevertheless, this endogenously bankruptcyfree situation is theoretically interesting.

\section{Numerical analysis}

\subsection{Probabilities and values}

In this section, we present numerical results. Throughout this section, parameter values we use are $f=1, r=0.01, \sigma=0.2$ and $\Delta=1$. Table 1 shows the default and postponement thresholds for each of the four cases: Model I $(N=0)$, Model II ( $N=1$ without resetting), Model III ( $N=1$ with resetting) and Model IV $(N=\infty)$. The tables at the top and at the bottom correspond to $\alpha=0.8$ and $\alpha=0.5$, respectively. Table 2 shows the 1-year default probability of an outstanding debt and the probabilities of bankruptcy within $1,2,5$ and 10 years when the current asset level is $A_{0}=1,1.5$ and 2. All the probabilities are under the risk-neutral probability measure. ${ }^{14}$ Figures 1 and 2 show the debt and stock values as a function of the current level of the firm asset for each of the four versions of our model and also for the "augmented" Merton model, the Merton model to which bankruptcy cost is added for comparison.

First we look at Model I. As is seen in Table 1, the default threshold, below which the stockholders choose not to repay debt, depends upon the size of $\alpha$, showing high

\footnotetext{
14 Instead, one can set a real drift term to the process of the firm asset value and compute 'actual' probabilities. As we focus on comparison across models in this paper, that is not necessary.
} 
Table 2 Default/bankruptcy probabilities

\begin{tabular}{|c|c|c|c|c|c|}
\hline & \multirow{2}{*}{$\begin{array}{l}\text { Probability } \\
\text { of default } \\
\text { in } 1 \text { year }\end{array}$} & \multicolumn{4}{|c|}{ Probability of bankruptcy within } \\
\hline & & 1 year & 2 years & 5 years & 10 years \\
\hline \multicolumn{6}{|l|}{ (i) $\alpha=0.8$} \\
\hline \multicolumn{6}{|l|}{$A_{0}=1$} \\
\hline Model I $(N=0)$ & 0.826 & 0.826 & 0.883 & 0.932 & 0.955 \\
\hline Model II $(N=1)$ & 0.826 & 0.825 & 0.883 & 0.932 & 0.955 \\
\hline Model III $(N=1)$ & 0.826 & 0.825 & 0.883 & 0.932 & 0.955 \\
\hline Model IV $(N=\infty)$ & 0.826 & 0.765 & 0.842 & 0.905 & 0.936 \\
\hline \multicolumn{6}{|l|}{$A_{0}=1.5$} \\
\hline Model I & 0.139 & 0.139 & 0.274 & 0.491 & 0.640 \\
\hline Model II & 0.139 & 0.139 & 0.274 & 0.491 & 0.640 \\
\hline Model III & 0.139 & 0.139 & 0.274 & 0.491 & 0.640 \\
\hline Model IV & 0.139 & 0.138 & 0.273 & 0.490 & 0.639 \\
\hline \multicolumn{6}{|l|}{$A_{0}=2$} \\
\hline Model I & 0.006 & 0.006 & 0.042 & 0.196 & 0.376 \\
\hline Model II & 0.006 & 0.006 & 0.042 & 0.196 & 0.376 \\
\hline Model III & 0.006 & 0.006 & 0.042 & 0.196 & 0.376 \\
\hline Model IV & 0.006 & 0.006 & 0.042 & 0.195 & 0.376 \\
\hline \multicolumn{6}{|l|}{ (ii) $\alpha=0.5$} \\
\hline \multicolumn{6}{|l|}{$A_{0}=1$} \\
\hline Model I $(N=0)$ & 0.996 & 0.996 & 0.998 & 0.999 & 0.999 \\
\hline Model II $(N=1)$ & 0.992 & 0.000 & 0.967 & 0.985 & 0.991 \\
\hline Model III $(N=1)$ & 0.987 & 0.000 & 0.939 & 0.962 & 0.976 \\
\hline Model IV $(N=\infty)$ & 0.520 & 0.000 & 0.000 & 0.000 & 0.000 \\
\hline \multicolumn{6}{|l|}{$A_{0}=1.5$} \\
\hline Model I & 0.729 & 0.729 & 0.813 & 0.888 & 0.926 \\
\hline Model II & 0.640 & 0.000 & 0.545 & 0.757 & 0.844 \\
\hline Model III & 0.572 & 0.000 & 0.446 & 0.649 & 0.765 \\
\hline Model IV & 0.024 & 0.000 & 0.000 & 0.000 & 0.000 \\
\hline \multicolumn{6}{|l|}{$A_{0}=2$} \\
\hline Model I & 0.204 & 0.204 & 0.352 & 0.559 & 0.693 \\
\hline Model II & 0.141 & 0.000 & 0.108 & 0.383 & 0.570 \\
\hline Model III & 0.105 & 0.000 & 0.072 & 0.298 & 0.489 \\
\hline Model IV & 0.000 & 0.000 & 0.000 & 0.000 & 0.000 \\
\hline
\end{tabular}

The tables present the probability of default in 1 year and the probabilities of bankruptcy within 1, 2, 5 and 10 years, for three different values for the current level of the firm asset $\left(A_{0}=1,1.5\right.$ and 2$)$. The size of $\alpha$ is 0.8 for (i) and 0.5 for (ii)

values for small $\alpha$. The reason is that when the firm repays debt partly by issuing a new debt, the value of this new debt is low if $\alpha$ is small. Therefore, the asset value itself must be higher to successfully raise the funds that are needed for repayment. 

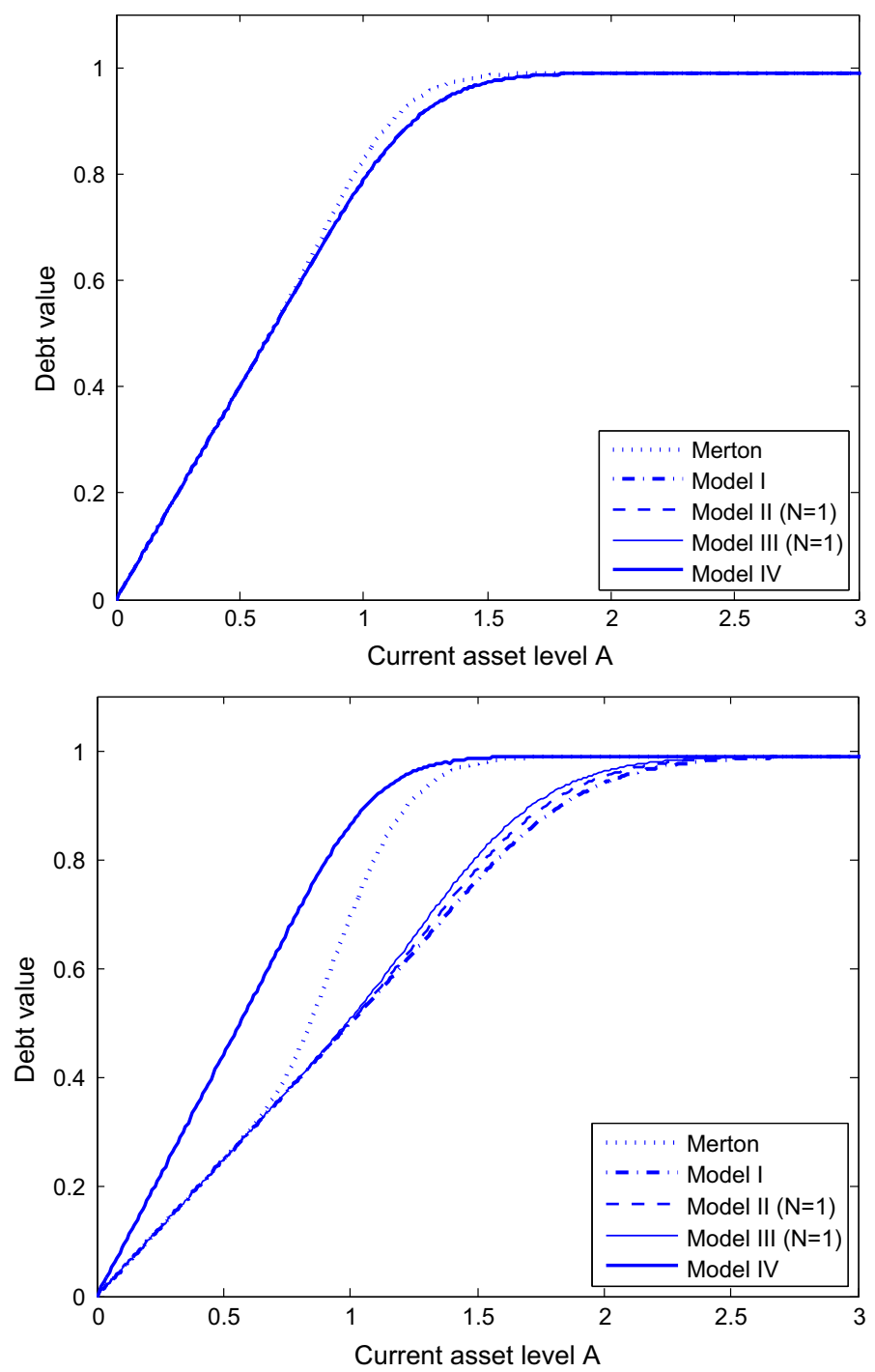

Fig. 1 Debt values. (i) $\alpha=0.8$ (top) and (ii) $\alpha=0.5$ (bottom) The figures give the debt values for $\alpha=0.8$ and 0.5 as a function of the current asset level

Next, comparing Fig. 1(i) and (ii) indicates that the debt value is lower when $\alpha$ is low. Similarly, comparing Fig. 2(i) and (ii) indicates that the stock value is also lower when $\alpha$ is low. The reason for the lower debt value is straightforward; low $\alpha$ means low bankruptcy/liquidation value and hence low debt value. On the other hand, the bankruptcy/liquidation value itself does not matter to the stockholders so the reason for the lower stock value is not so obvious. The reason is that low $\alpha$ leads to high default threshold, which in turn makes future bankruptcy more likely. In comparison with the Merton model, our $N=0$ model shows lower stock values as are seen in Fig. 2(i)-(ii). The Merton model, while we augment it with the same bankruptcy cost, 

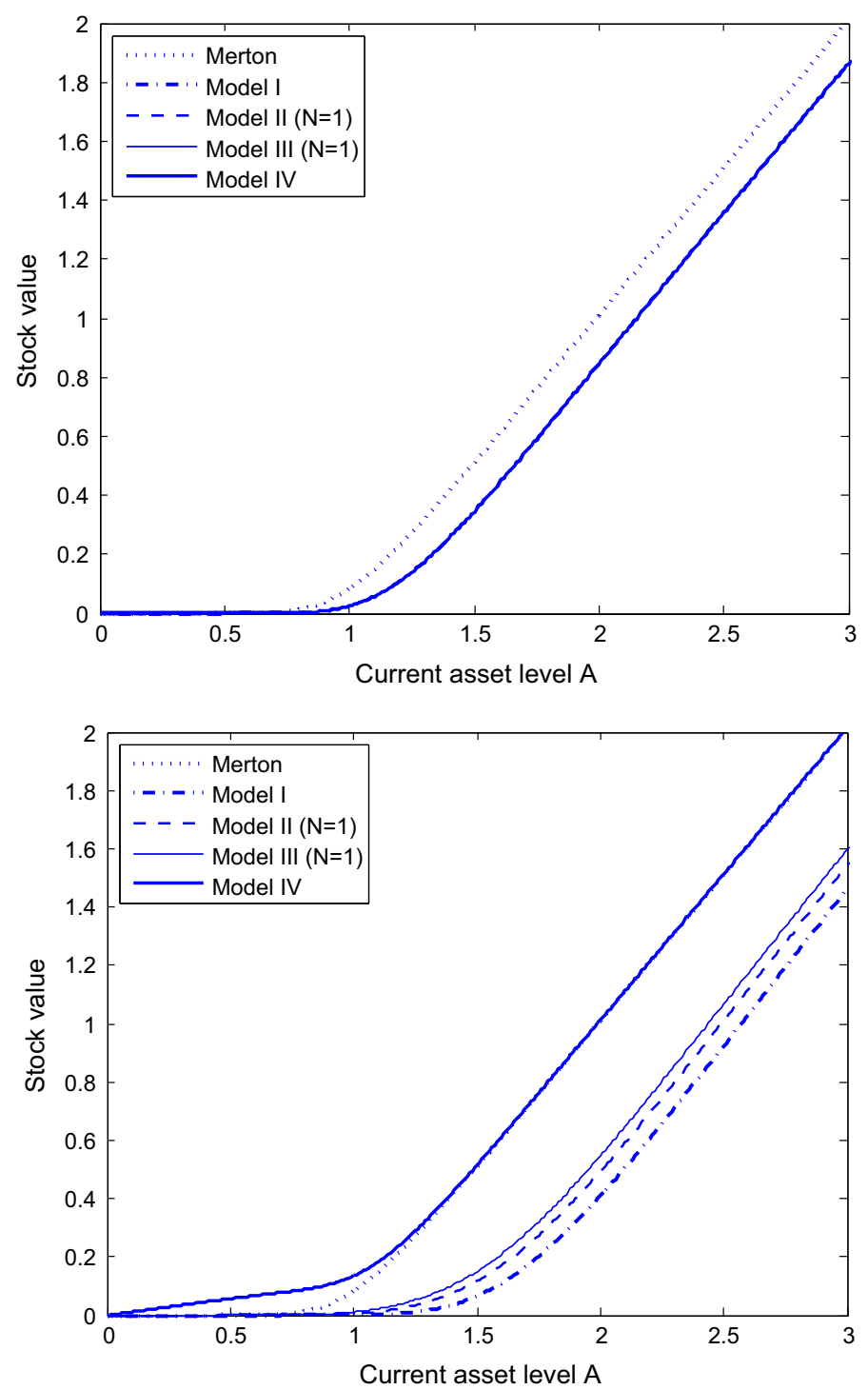

Fig. 2 Stock values. (i) $\alpha=0.8$ (top) and (ii) $\alpha=0.5$ (bottom) The figures give the stock values for $\alpha=0.8$ and 0.5 as a function of the current asset level

does not involve refinancing so the default threshold still remains $f$. In contrast, the default threshold in our model exceeds $f$ and hence default/bankruptcy becomes more likely, which in turn lowers the stock value compared with the Merton model.

Next we look into Model II $(N=1)$. As is seen by comparing Models I and II in Table 2(i) and Fig. 1(i) and 2(i), the effect of allowing for maturity extension on debt/stock values and default/bankruptcy probabilities is almost zero when the asset recovery rate is high $(\alpha=0.8)$. If $\alpha$ is high, liquidation on default is not so costly and is attractive most of the time. Table 1(i) indicates that the creditor chooses to 
exercise the postponement option only when the firm's asset value is extremely low. In such situations, however, the debt and stock values are very low anyway. In contrast, when $\alpha$ is low, introducing the postponement option has a large impact. Figures 1(ii) and 2(ii) show that the debt and stock values of Model II are significantly higher than those of Model I. Also, Table 2(ii) shows that for Model II, the probabilities of bankruptcy within 1, 2, 5 and 10 years are lower than for Model I. As is seen in Table 1(ii), when $\alpha$ is as low as 0.5 , the postponement threshold is higher than the default threshold. Therefore, whenever default occurs, the creditor routinely postpones the repayment, which is the case described in Proposition 2.3. This simply makes the 1-year bankruptcy probability zero. The mechanism for the reduction of the 2-year bankruptcy probability is more complex; it is not only because the probability of the first-year bankruptcy is zero but also because the postponement option has made the default threshold lower. The introduction of postponement into the model lowers future bankruptcy risk and raises the present debt and stock values. Since our model assumes refinancing that uses these debt and stock, the increase of their values makes the default risk (i.e., the default threshold) go down. ${ }^{15}$ If the firm does not default for the first year, then the creditor keeps the postponement option for the second year. In this way, the second-year bankruptcy probability further goes down.

Now we examine Model III $(N=1)$ in comparison with Model II $(N=1)$ when $\alpha=0.5$, to see the impact of the reset assumption. First, Table 1(ii) shows a lower default threshold for Model III, and correspondingly, Table 2(ii) shows a smaller 1year default probability for Model III. When the present asset values are 1, 1.5 and 2, the 1-year default probabilities for Model II are 0.992, 0.640 and 0.141, respectively, while they are $0.987,0.572$ and 0.105 for Model III. The reset assumption directly lowers future bankruptcy risk, raises the debt and stock values, making refinancing easier, and lowers the default threshold. Moreover, 2-year bankruptcy probabilities of the model with reset, $(0.939,0.446,0.072)$ are lower than those of the model without it $(0.967,0.545,0.108)$. This is not only because the 1 -year default risk is lower in the former model, but also because of the following reason. Once the creditor exercises the postponement option in the $N=1$ model without reset, then it becomes the $N=0$ model, and the default threshold increases from $\bar{A}^{1}$ to $\bar{A}^{0}$. On the other hand, as we saw in Proposition 2.4, in the model with resetting, even if the creditor exercises the postponement option, the default threshold does not change. So the probability of bankruptcy conditional on one default in the past is lower in the model with resetting. At first look, one may expect that whether there is resetting or not does not matter much to short-term risk of default or bankruptcy. The fact is that the reduction in the long-term bankruptcy risk by reset assumption raises the present stock value, making refinancing easier, and in turn reduces the short-term default/bankruptcy risk.

The above effect is even bigger for larger $N$. In the extreme case of $N=\infty$ and $\alpha=0.5$, that is the endogenously bankruptcy-free case of Proposition 2.5 , the bankruptcy probability is zero and debt/stock values are even higher. Also, the default threshold coincides with the principal of the debt. These facts make the model resemble the Merton model at high asset values. At a low asset level, our $N=\infty$ model gives a

15 The causality explained here is for the sake of intuition. In reality, everything is determined simultaneously as the solution to the system of integral equations. 
higher stock value than Merton because the payoff to the stock holder when the asset value is below the debt principal is zero in the Merton model but not zero in our model.

\subsection{Asset volatility and stock/debt values}

In this subsection, we investigate the relation between the volatility of the firm asset, $\sigma$, and the debt and stock values. First we look at the stock value. In the standard Merton model, the stock price is the Black-Scholes price of an European call option for which the underlying asset is the firm asset. Therefore, high asset volatility yields high stock price. In particular, when the asset value is around the at-the-money level, high volatility leads to high stock value because the potential gain to stockholders becomes large while the potential loss is bounded. When the asset level is deep-inthe-money, the increase in the asset volatility affects the stockholders' potential gains and losses in a similar way, leading to very little effect on the stock value.

The effect of change in $\sigma$ on the stock value in our model is presented in Fig. 3 (top). In this figure, the vertical axis is the partial derivative of the stock value with respect to $\sigma$ evaluated at $\sigma=0.2$, and the horizontal axis is the current level of the firm asset. ${ }^{16}$ Because qualitative features are similar across Models I-III, we focus on Model I. In the low asset region, the derivative is always positive, indicating that the increase in $\sigma$ leads to the increase in the stock value. That is, when the firm asset level is relatively low, the aforementioned positive effect of volatility on the stock value in the standard Merton model is also present in our model of refinancing. The effect is larger due to the refinancing multiplier between the stock value and the default probability.

In the high asset region, on the other hand, our model exhibits a negative effect of $\sigma$ on the stock price, unlike the Merton model. This is because our model with refinancing is different from Merton in that the default threshold is no longer constant but depends on the magnitude of asset volatility. While the increase in the asset volatility has the direct positive effect on stock price that is present in the Merton model, it also lowers the debt value around the default threshold and hence increases the default threshold, which turns into a negative effect on the stock value. When the asset value is deepin-the-money, the latter effect dominates the former, and thus high $\sigma$ lowers the stock value.

Next we examine the relation between $\sigma$ and the debt value. In the standard Merton model without bankruptcy cost, the debt price resembles the short-selling of the (Black-Scholes) European put option for which the underlying asset is the firm asset. Therefore, in the standard Merton model, high asset volatility always yields low debt price. With the presence of bankruptcy cost, however, that is not always the case. In particular, in the Merton model augmented with bankruptcy $\cos t \alpha<1$, one can show that there is a cutoff asset level $\hat{A}$ such that $\partial F(A) / \partial \sigma>(<) 0$ if $A<(>) \hat{A}$. That is, if the current asset level is sufficiently low, high volatility yields high debt value. This is due to the discontinuity that $\alpha$ causes. When the asset level is in the low region so the costly bankruptcy is likely, the benefit of high volatility is larger than the down-

\footnotetext{
16 The partial derivatives are computed numerically: the equilibrium stock and debt values are computed for $\sigma=0.195$ and $\sigma=0.205$, and the rate of change is computed.
} 

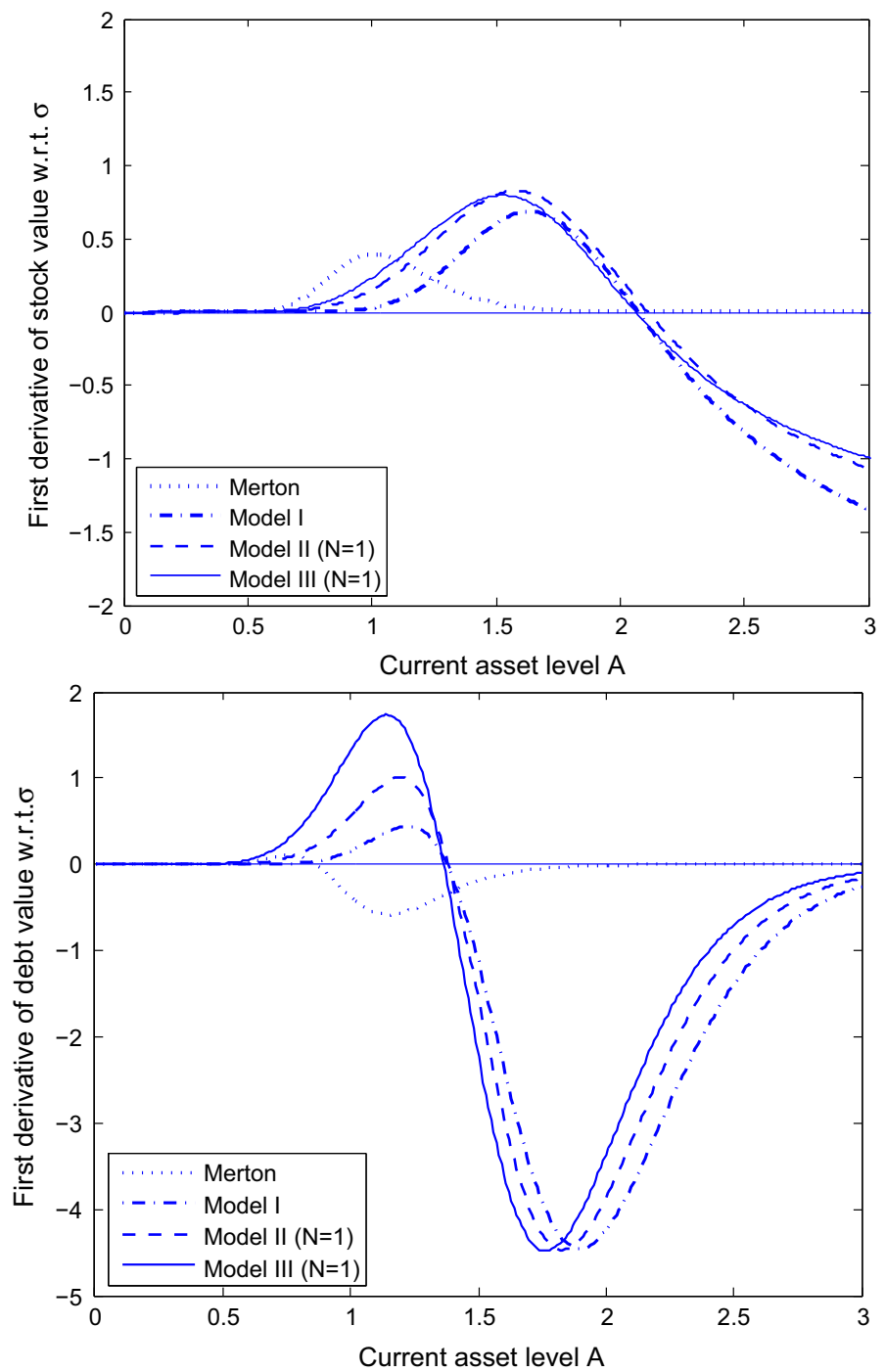

Fig. 3 Partial derivative of stock and debt values with respect to $\sigma$ Numerical first-derivatives of the stock (top) and debt (bottom) values with respect to $\sigma$ evaluated at $\sigma=0.2$. The $x$-axis is the current asset value. $(\alpha=0.5)$

side of high volatility because high volatility means a good chance of avoiding costly bankruptcy. This is a direct channel through which the increase in $\sigma$ has a positive effect on the debt value for the low asset region.

In the model with refinancing (Model I), there is also a rather indirect channel through which higher volatility can lead to higher debt value. As we saw, higher volatility leads to higher stock value when the asset level is relatively low. In the model with refinancing, higher stock value implies a better chance of successful refinancing, lowering the default probability and increasing the debt value. Moreover, because the 
positive effect of high volatility on the stock value in the model of refinancing is even larger when there is a possibility of maturity extension, so is its indirect impact on the debt value [see Model II and III in Fig. 3 (bottom)].

One common statement in the field of corporate finance is that stockholders are risk-takers as residual claimants while debt holders avert risk and prefer safe business. This is not necessarily the case in models with bankruptcy cost and refinancing. When the asset value is high, stockholders may prefer lower business risk to avoid bad refinancing conditions. When the asset value is relatively low, creditors may prefer higher business risk because that raises the stock value, makes successful refinancing more likely, and lowers the default/bankruptcy probability.

\section{Conclusion}

In this article we study a structural model of corporate credit risk in which the debt repayment is made by refinancing. We further extend the model by incorporating the creditor's option to extend the date of debt maturity, partly motivated by the observation that under Japan's main creditor system, the main creditor of a firm often postpones the firm's repayment on default. In our refinancing model without such a postponement option, the lower asset recovery rate $\alpha$ leads to higher bankruptcy risk and lower debt/stock values. While introducing the creditor's postponement option has a very limited effect when $\alpha$ is high, it reduces bankruptcy risk and raises the debt/stock values significantly when $\alpha$ is low.

Our model sheds light on some easily overlooked channels through which the possibility of flexible maturity extension affects the default and bankruptcy probabilities of a firm. One such channel is a multiplier effect of refinancing on default probabilities; an initial reduction of bankruptcy risk by the possibility of flexible maturity extension raises debt/stock prices, facilitates refinancing, and further pushes down the default/bankruptcy risk. Another channel is the effect of reduction in the long-run bankruptcy risk on the short-run bankruptcy risk. This effect was found in the comparison of Models II and III. That is, an arrangement that seemingly decreases only the long-run risk can also lower the short-run risk because the former affects the current security prices, which matter to the latter in the process of refinancing.

In this article we assume that the amount of debt is exogenously fixed. In a more realistic environment, it should be the decision of a firm that solves the optimal capital structure problem facing advantages of debt (e.g. tax benefit) and disadvantages of debt (e.g. cost of bankruptcy). Whether our existence proof can be extended to such an environment is not known. Nevertheless, our simple framework serves a basis for the argument that both refinancing and renegotiation such as maturity extension are important to corporate risk analysis.

Open Access This article is distributed under the terms of the Creative Commons Attribution 4.0 International License (http://creativecommons.org/licenses/by/4.0/), which permits unrestricted use, distribution, and reproduction in any medium, provided you give appropriate credit to the original author(s) and the source, provide a link to the Creative Commons license, and indicate if changes were made. 


\section{Appendix: Proofs}

First we show two preliminary lemmas and then prove propositions in order.

Lemma 4.1 (Extension of the Blackwell sufficient condition for contractions) Let $X$ be a subset of the Euclidean space $\mathbb{R}^{D}$ and $\psi$ be a real-valued function on $X$. Let $V$ be the set of real-valued continuous functions on $X$ whose distance from $\psi$ is finite, where the metric between two functions is given by $d(g, k) \equiv \sup _{A \in X}|g(A)-k(A)|$. Also, the metric between two vectors of $N$ functions, $G=\left(g^{1}, \ldots, g^{N}\right) \in V^{N}$ and $K=\left(k^{1}, \ldots, k^{N}\right) \in V^{N}$, is defined as $d_{\infty}(G, K) \equiv \max _{M \in\{1, \ldots, N\}} d\left(g^{M}, k^{M}\right) . V^{N}$ is a complete metric space. We say $G \leq K$ if $g^{M}(A) \leq k^{M}(A)$ for all $A \in X$ and all $M \in\{1, \ldots, N\}$. Consider for each $M=1, \ldots, N$, a mapping $T_{M}: V^{N} \rightarrow V$ and let $T=\left(T_{1}, \ldots, T_{N}\right)$. Suppose that $T: V^{N} \rightarrow V^{N}$ satisfies the following;

(i) (monotonicity): $G \leq K \Rightarrow T G \leq T K$; and

(ii) (discounting): for all $G \in V^{N}$ and $a=\left(a_{1}, \ldots, a_{N}\right) \in \mathbb{R}_{++}^{N}$, there exists $\beta \in$ $(0,1)$ such that $T(G+a) \leq T G+\beta\left(\max _{M} a_{M}\right)$ e, where $e \equiv(1,1, \ldots, 1)^{\prime}$.

Then $T$ is a contraction with modulus $\beta$.

Proof For any $G, K \in V^{N}$ and $M=1, \ldots, N$, we have $g^{M}=k^{M}+\left(g^{M}-k^{M}\right) \leq$ $k^{M}+d\left(g^{M}, k^{M}\right)$. So,

$$
T\left(\begin{array}{c}
g^{1} \\
\vdots \\
g^{N}
\end{array}\right) \leq T\left(\begin{array}{c}
k^{1}+d\left(g^{1}, k^{1}\right) \\
\vdots \\
k^{N}+d\left(g^{N}, k^{N}\right)
\end{array}\right) \leq T\left(\begin{array}{c}
k^{1} \\
\vdots \\
k^{N}
\end{array}\right)+\beta \max _{j} d\left(g^{j}, k^{j}\right)\left(\begin{array}{c}
1 \\
\vdots \\
1
\end{array}\right)
$$

where the first inequality comes from monotonicity and the second from discounting. Hence for each $M$, we have $T_{M} G-T_{M} K \leq \beta \max _{j} d\left(g^{j}, k^{j}\right)$. Switching the role of $G$ and $K$ gives $T_{M} K-T_{M} G \leq \beta \max _{j} d\left(g^{j}, k^{j}\right)$. Thus $d\left(T_{M} G, T_{M} K\right) \leq$ $\beta \max _{j} d\left(g^{j}, k^{j}\right)$ for all $M$. This implies $\max _{M} d\left(T_{M} G, T_{M} K\right) \leq \beta \max _{j} d\left(g^{j}, k^{j}\right)$, or $d_{\infty}(T G, T K) \leq \beta d_{\infty}(G, K)$.

Lemma 4.2 (Comparative statics of the fixed point of parameterized contraction) Let $\left(V^{N}, d_{\infty}\right)$ be the metric space defined in Lemma 4.1. Let $T^{\theta}: V^{N} \rightarrow V^{N}$, where $\theta \in \Theta \subset \mathbb{R}_{+}$is a parameter. Assume that for each $\theta, T^{\theta}$ is a contraction that satisfies Blackwell's monotonicity condition. Denote its fixed point as $H(\cdot ; \theta)=$ $\left(h^{1}(\cdot ; \theta), \ldots, h^{N}(\cdot ; \theta)\right)$. Assume that $T^{\theta}$ satisfies the parameter-monotonicity: for any $\theta_{1}<\theta_{2}$ and $G \in V^{N}$,

$$
\left(T^{\theta_{1}} G\right)(x)<\left(T^{\theta_{2}} G\right)(x), \forall x,
$$

where the inequality is element-wise. Then $H(\cdot ; \theta)$ is strictly increasing in $\theta$, namely,

$$
H\left(x ; \theta_{1}\right)<H\left(x ; \theta_{2}\right), \forall \theta_{1}<\theta_{2}, \forall x .
$$


Proof Choose an arbitrary $K \in V^{N}$. Define sequences of functions by $H_{0}=$ $G_{0}=K, H_{n}=T^{\theta_{1}} H_{n-1}$ and $G_{n}=T^{\theta_{2}} G_{n-1}, n=1,2, \ldots$ Then by parametermonotonicity of $T^{\theta}$, we have $H_{1} \leq G_{1}$. Suppose $H_{n} \leq G_{n}$. Then $H_{n+1}=T^{\theta_{1}} H_{n} \leq$ $T^{\theta_{1}} G_{n}<T^{\theta_{2}} G_{n}=G_{n+1}$, where the first inequality comes from Blackwell's monotonicity and the second from the parameter-monotonicity. Since $H_{n} \rightarrow H\left(\cdot ; \theta_{1}\right)$ and $G_{n} \rightarrow H\left(\cdot ; \theta_{2}\right)$, we have $H\left(\cdot ; \theta_{1}\right) \leq H\left(\cdot ; \theta_{2}\right)$. To show strict inequality, note that $H\left(\cdot ; \theta_{1}\right)=T^{\theta_{1}} H\left(\cdot ; \theta_{1}\right) \leq T^{\theta_{1}} H\left(\cdot ; \theta_{2}\right)<T^{\theta_{2}} H\left(\cdot ; \theta_{2}\right)=H\left(\cdot ; \theta_{2}\right)$.

Proof of Proposition 2.1 Define the total market value of the firm as $H^{0}(\cdot) \equiv F^{0}(\cdot)+$ $S^{0}(\cdot)$. Summing up (3) and (4) gives

$$
H^{0}(A)=e^{-r \Delta} E_{\tilde{A} \mid A}\left[\mathbf{1}_{\left\{\tilde{A} \geq \bar{A}^{0}\right\}} H^{0}(\tilde{A})+\mathbf{1}_{\left\{\tilde{A}<\bar{A}^{0}\right\}} \alpha \tilde{A}\right]
$$

and (5) becomes

$$
H^{0}\left(\bar{A}^{0}\right)=f
$$

To show the existence of the solution of our interest, define a mapping by the right side of (22). Formally, define a complete metric space $V$ as in Lemma 4.1 with $X=\mathbb{R}_{++}$and $\psi(A)=A$. (That is, $\psi$ is identity function on $\mathbb{R}_{++}$.) ${ }^{17}$ Define a mapping $T_{0}^{\bar{A}}: V \rightarrow V$ by replacing $\bar{A}^{0}$ in the r.h.s. of (22) with an arbitrary threshold $\bar{A}$ :

$$
\left(T_{0}^{\bar{A}} g\right)(A) \equiv e^{-r \Delta} E_{\tilde{A} \mid A}\left[\mathbf{1}_{\{\tilde{A} \geq \bar{A}\}} g(\tilde{A})+\mathbf{1}_{\{\tilde{A}<\bar{A}\}} \alpha \tilde{A}\right] .
$$

Indeed, $T_{0}^{\bar{A}} g \in V$ is true as we can show that for any $g \in V$, the distance between $T_{0}^{\bar{A}} g$ and the identity function $\psi(A)=A$ is finite: that is,

$$
\begin{aligned}
\left(T_{0}^{\bar{A}} g\right)(A)-A & =e^{-r \Delta} E_{\tilde{A} \mid A}\left[\mathbf{1}_{\{\tilde{A} \geq \bar{A}\}} g(\tilde{A})+\mathbf{1}_{\{\tilde{A}<\bar{A}\}} \alpha \tilde{A}\right]-A \\
& =e^{-r \Delta} E_{\tilde{A} \mid A}\left[\mathbf{1}_{\{\tilde{A} \geq \bar{A}\}} g(\tilde{A})+\mathbf{1}_{\{\tilde{A}<\bar{A}\}} \alpha \tilde{A}\right]-e^{-r \Delta} E_{\tilde{A} \mid A}[\tilde{A}] \\
& =e^{-r \Delta} E_{\tilde{A} \mid A}\left[\mathbf{1}_{\{\tilde{A} \geq \bar{A}\}}(g(\tilde{A})-\tilde{A})+\mathbf{1}_{\{\tilde{A}<\bar{A}\}}(\alpha \tilde{A}-\tilde{A})\right] \\
& =e^{-r \Delta} E_{\tilde{A} \mid A}\left[\mathbf{1}_{\{\tilde{A} \geq \bar{A}\}}(g(\tilde{A})-\tilde{A})\right]-(1-\alpha) e^{-r \Delta} E_{\tilde{A} \mid A}\left[\mathbf{1}_{\{\tilde{A}<\bar{A}\}} \tilde{A}\right],
\end{aligned}
$$

where the first term of the last line is finite because $g \in V$, so is the second term because $0 \leq E\left[\mathbf{1}_{\{\tilde{A}<\bar{A}\}} \tilde{A}\right] \leq \bar{A}$. We show $T_{0}^{\bar{A}}$ is a contraction by approaching Blackwell's sufficient condition for contraction. For monotonicity, suppose $h(A) \leq g(A), \forall A>0$.

17 Since the expectation is in terms of the risk-neutral measure (1), $e^{-r \Delta} E_{\tilde{A} \mid A}[\tilde{A}]=A$ holds. Therefore, Eq. (22) has an obvious solution $\alpha A$, which is not in space $V$. 
Then

$$
\begin{aligned}
\left(T_{0}^{\bar{A}} h\right)(A) & =e^{-r \Delta} E_{\tilde{A} \mid A}\left[\mathbf{1}_{\{\tilde{A} \geq \bar{A}\}} h(\tilde{A})+\mathbf{1}_{\{\tilde{A}<\bar{A}\}} \alpha \tilde{A}\right] \\
& \leq e^{-r \Delta} E_{\tilde{A} \mid A}\left[\mathbf{1}_{\{\tilde{A} \geq \bar{A}\}} g(\tilde{A})+\mathbf{1}_{\{\tilde{A}<\bar{A}\}} \alpha \tilde{A}\right]=\left(T_{0}^{\bar{A}} g\right)(A), \quad \forall A>0 .
\end{aligned}
$$

For discounting, suppose $g \in V$ and $a>0$. Then

$$
\begin{aligned}
\left(T_{0}^{\bar{A}}(g+a)\right)(A) & =e^{-r \Delta} E_{\tilde{A} \mid A}\left[\mathbf{1}_{\{\tilde{A} \geq \bar{A}\}}(g(\tilde{A})+a)+\mathbf{1}_{\{\tilde{A}<\bar{A}\}} \alpha \tilde{A}\right] \\
& =e^{-r \Delta} E_{\tilde{A} \mid A}\left[\mathbf{1}_{\{\tilde{A} \geq \bar{A}\}} g(\tilde{A})+\mathbf{1}_{\{\tilde{A}<\bar{A}\}} \alpha \tilde{A}\right]+e^{-r \Delta} a E_{\tilde{A} \mid A}\left[\mathbf{1}_{\{\tilde{A} \geq \bar{A}\}}\right] \\
& \leq\left(T_{0}^{\bar{A}} g\right)(A)+\beta a
\end{aligned}
$$

where $\beta \equiv e^{-r \Delta} E_{\tilde{A} \mid A}\left[\mathbf{1}_{\{\tilde{A} \geq \bar{A}\}}\right]<1$. Therefore $T_{0}^{\bar{A}}$ is a contraction on $V$, and by the contraction mapping theorem, it has a unique fixed point, denoted $h^{0}(\cdot ; \bar{A}) \in V$. (Note that the solution to $(22)$ is a special case and $H^{0}(\cdot)=h^{0}\left(\cdot ; \bar{A}^{0}\right)$.)

Now we show that $h^{0}(A ; \bar{A})$ is strictly increasing in $A$ and satisfies $\alpha A<$ $h^{0}(A ; \bar{A})<A$ for all $A>0$ by approaching Corollary 1, p52 of Stokey Lucas Prescott. Define sets

$$
\begin{aligned}
W & \equiv\{g \in V: \text { nondecreasing and } \alpha A \leq g(A) \leq A \text { for all } A>0\} ; \text { and } \\
W^{\prime} & \equiv\{g \in V: \text { strictly increasing and } \alpha A<g(A)<A \text { for all } A>0\}
\end{aligned}
$$

$W$ is a closed subset of $V$ and the Corollary of SLP implies that if $T_{0}^{\bar{A}}(W) \subset W^{\prime}$ then $h^{0}(\cdot ; \bar{A}) \in W^{\prime}$. Suppose $g \in W$ and $0<A<A^{\prime}$. Then

$$
\begin{aligned}
\left(T_{0}^{\bar{A}} g\right)(A) & =e^{-r \Delta} E_{\tilde{A} \mid A}\left[\mathbf{1}_{\{\tilde{A} \geq \bar{A}\}} g(\tilde{A})+\mathbf{1}_{\{\tilde{A}<\bar{A}\}} \alpha \tilde{A}\right] \\
& <e^{-r \Delta} E_{\tilde{A} \mid A^{\prime}}\left[\mathbf{1}_{\{\tilde{A} \geq \bar{A}\}} g(\tilde{A})+\mathbf{1}_{\{\tilde{A}<\bar{A}\}} \alpha \tilde{A}\right]=\left(T_{0}^{\bar{A}} g\right)\left(A^{\prime}\right),
\end{aligned}
$$

where the inequality results from the fact that $\tilde{A}$ is log-normally distributed conditional on $A$ (or $A^{\prime}$ ) and that $g \in W$ implies the inside of the expectation operator is nondecreasing, non-constant function. Also we have

$$
\begin{aligned}
\left(T_{0}^{\bar{A}} g\right)(A) & =e^{-r \Delta} E_{\tilde{A} \mid A}\left[\mathbf{1}_{\{\tilde{A} \geq \bar{A}\}} g(\tilde{A})+\mathbf{1}_{\{\tilde{A}<\bar{A}\}} \alpha \tilde{A}\right] \\
& \leq e^{-r \Delta} E_{\tilde{A} \mid A}\left[\mathbf{1}_{\{\tilde{A} \geq \bar{A}\}} \tilde{A}+\mathbf{1}_{\{\tilde{A}<\bar{A}\}} \alpha \tilde{A}\right]<e^{-r \Delta} E_{\tilde{A} \mid A}[\tilde{A}]=A,
\end{aligned}
$$

and,

$$
\begin{aligned}
\left(T_{0}^{\bar{A}} g\right)(A) & =e^{-r \Delta} E_{\tilde{A} \mid A}\left[\mathbf{1}_{\{\tilde{A} \geq \bar{A}\}} g(\tilde{A})+\mathbf{1}_{\{\tilde{A}<\bar{A}\}} \alpha \tilde{A}\right] \\
& >e^{-r \Delta} E_{\tilde{A} \mid A}\left[\mathbf{1}_{\{\tilde{A} \geq \bar{A}\}} \alpha \tilde{A}+\mathbf{1}_{\{\tilde{A}<\bar{A}\}} \alpha \tilde{A}\right]=\alpha A,
\end{aligned}
$$


where the inequality is because $g \in W \subset V$ implies $g(\tilde{A})>\alpha \tilde{A}$ for sufficiently large $\tilde{A}$ 's. Hence we have $T_{0}^{\bar{A}}(W) \subset W^{\prime}$. Thus $h^{0}(\cdot ; \bar{A}) \in W^{\prime}$.

As a special case, we have $\alpha \dot{\bar{A}}<h^{0}(\bar{A} ; \bar{A})<\bar{A}$. We show that $h^{0}(\bar{A} ; \bar{A})$ is continuous as a function of $\bar{A}$. First, regard $h^{0}(\cdot ; \bar{A})$ as a mapping that maps $\bar{A}>0$ to $h^{0}(\cdot ; \bar{A}) \in W^{\prime}$. It is not hard to show that this mapping is continuous, or $\bar{A}_{n} \rightarrow \bar{A} \Rightarrow$ $\sup _{x>0}\left|h^{0}\left(x ; \bar{A}_{n}\right)-h^{0}(x ; \bar{A})\right| \rightarrow 0$, so we have

$$
\begin{aligned}
\left|h^{0}\left(\bar{A}_{n} ; \bar{A}_{n}\right)-h^{0}(\bar{A} ; \bar{A})\right| & \leq\left|h^{0}\left(\bar{A}_{n} ; \bar{A}_{n}\right)-h^{0}\left(\bar{A}_{n} ; \bar{A}\right)\right|+\left|h^{0}\left(\bar{A}_{n} ; \bar{A}\right)-h^{0}(\bar{A} ; \bar{A})\right| \\
& \leq \sup _{x>0}\left|h^{0}\left(x ; \bar{A}_{n}\right)-h^{0}(x ; \bar{A})\right|+\left|h^{0}\left(\bar{A}_{n} ; \bar{A}\right)-h^{0}(\bar{A} ; \bar{A})\right| \rightarrow 0
\end{aligned}
$$

as $\bar{A}_{n} \rightarrow \bar{A}$ (the second term goes to zero because $h^{0}(\cdot ; \bar{A}) \in V$ ). Therefore $h^{0}(\bar{A} ; \bar{A})$ is continuous as a function of $\bar{A}$. Hence a variation of the intermediate value theorem implies that there exists $\bar{A}^{*}$ such that $h^{0}\left(\bar{A}^{*} ; \bar{A}^{*}\right)=f$, and this $\bar{A}^{*}$ satisfies $\alpha \bar{A}^{*}<$ $f<\bar{A}^{*}$. This concludes the existence of $\bar{A}^{0}$ together with $H^{0}(\cdot) \equiv h^{0}\left(\cdot ; \bar{A}^{0}\right)$. This gives $F^{0}(\cdot)$ by $(6)$ and $S^{0}(\cdot)=H^{0}(\cdot)-F^{0}(\cdot)$.

Proof of Proposition 2.2 It suffices to show the following general statement. Consider Eqs. (6)-(7) with $\bar{A}^{0}$ replaced by an arbitrary threshold $\bar{B}^{0}$ :

$$
\begin{aligned}
F^{0}\left(A ; \bar{B}^{0}\right) & =\alpha A \Phi\left(-x_{1}\right)+f e^{-r \Delta} \Phi\left(x_{2}\right), \text { where } \\
x_{1}, x_{2} & =\frac{\log \left(A / \bar{B}^{0}\right)+\left(r \pm \sigma^{2} / 2\right) \Delta}{\sigma \sqrt{\Delta}} .
\end{aligned}
$$

Then there exists $\underline{B}^{1}$ such that $F^{0}\left(\tilde{A} ; \bar{B}^{0}\right)-\alpha \tilde{A} \gtreqless 0 \Leftrightarrow \tilde{A} \lesseqgtr \underline{B}^{1}, \forall \tilde{A}$.

For proof, define function $G(A) \equiv F^{0}\left(A ; \bar{B}^{0}\right)-\alpha A$. We have $\lim _{A \rightarrow 0} G(A)=0$ and $\lim _{A \rightarrow \infty} G(A)=-\infty$. Let $\phi(\cdot)$ be the density function of $\Phi(\cdot)$. From (25)-(26), we have

$$
G^{\prime}(A)=\alpha \Phi\left(-x_{1}\right)-\alpha A \phi\left(-x_{1}\right) \frac{\partial x_{1}}{\partial A}+f e^{-r \Delta} \phi\left(x_{2}\right) \frac{\partial x_{2}}{\partial A}-\alpha .
$$

Definition of $x_{1}$ and $x_{2}$ implies $\partial x_{1} / \partial A=\partial x_{2} / \partial A=(A \sigma \sqrt{\Delta})^{-1}, x_{2}=x_{1}-$ $\sigma \sqrt{\Delta}$, and $\phi\left(x_{2}\right)=\phi\left(x_{1}\right) e^{r \Delta} A / \bar{B}^{0}$. Substituting these into (27) yields

$$
G^{\prime}(A)=\left(\Phi\left(-x_{1}\right)-1\right) \alpha+\frac{1}{\bar{B}^{0}} \frac{\phi\left(x_{1}\right)}{\sigma \sqrt{\Delta}}\left(f-\alpha \bar{B}^{0}\right) .
$$

Note that $\lim _{A \rightarrow 0} G^{\prime}(A)=0$ and $\lim _{A \rightarrow \infty} G^{\prime}(A)=-\alpha$. Also, $\phi^{\prime}(x)=(-x) \phi(x)$ implies $G^{\prime \prime}(A)=\frac{\phi\left(x_{1}\right)}{A \sigma \sqrt{\Delta}}\left(-\alpha-x_{1} K\right)$, where $K \equiv \frac{1}{\sigma \sqrt{\Delta}}\left(\frac{f}{\bar{B}^{0}}-\alpha\right)>0$. Let $A^{\prime}$ be the asset level such that $x_{1}=-\alpha / K$. Then $A<A^{\prime} \Leftrightarrow x_{1}<-\alpha / K \Leftrightarrow G^{\prime \prime}(A)>0$. This together with $\lim _{A \rightarrow 0} G^{\prime}(A)=0$ and $\lim _{A \rightarrow \infty} G^{\prime}(A)=-\alpha$ imply that there exists $A^{\prime \prime}>A^{\prime}$ such that $A<A^{\prime \prime} \Leftrightarrow G^{\prime}(A)>0, A=A^{\prime \prime} \Leftrightarrow G^{\prime}(A)=0$ and $A>A^{\prime \prime} \Leftrightarrow$ $G^{\prime}(A)<0$. But then this together with $\lim _{A \rightarrow 0} G(A)=0$ and $\lim _{A \rightarrow \infty} G(A)=-\infty$ 
implies that there exists $A^{\prime \prime \prime}>A^{\prime \prime}$ such that $A<A^{\prime \prime \prime} \Leftrightarrow G(A)>0, A=A^{\prime \prime \prime} \Leftrightarrow$ $G(A)=0$ and $A>A^{\prime \prime \prime} \Leftrightarrow G(A)<0$. Thus $\underline{B}^{1} \equiv A^{\prime \prime \prime}$ concludes the proof.

Proof of Proposition 2.3 Defining $H^{N}(\cdot) \equiv F^{N}(\cdot)+S^{N}(\cdot)$ for all $N \in \mathbb{N}$ and summing up (8) and (9) lead to

$$
\begin{aligned}
H^{N}(A)= & e^{-r \Delta} E_{\tilde{A} \mid A}\left[\mathbf{1}_{\left\{\tilde{A} \geq \bar{A}^{N}\right\}} H^{N}(\tilde{A})\right. \\
& \left.+\mathbf{1}_{\left\{\tilde{A}<\bar{A}^{N}\right\}}\left(\mathbf{1}_{\left\{\alpha \tilde{A} \geq F^{N-1}(\tilde{A})\right\}} \alpha \tilde{A}+\mathbf{1}_{\left\{\alpha \tilde{A}<F^{N-1}(\tilde{A})\right\}} H^{N-1}(\tilde{A})\right)\right] .
\end{aligned}
$$

Also, (10) becomes

$$
H^{N}\left(\bar{A}^{N}\right)=f
$$

$F^{0}(\cdot), H^{0}(\cdot)$ and $\bar{A}^{0}$ are already given in Model I. Given $F^{N-1}$ and $H^{N-1}$, define a mapping $T_{N}^{\bar{A}}: V \rightarrow V$ by the r.h.s of (28) with $\bar{A}^{N}$ replaced by an arbitrary threshold $\bar{A}$;

$$
\left(T_{N}^{\bar{A}} g\right)(A) \equiv e^{-r \Delta} E_{\tilde{A} \mid A}\left[\mathbf{1}_{\{\tilde{A} \geq \bar{A}\}} g(\tilde{A})+\mathbf{1}_{\{\tilde{A}<\bar{A}\}} L^{N-1}(\tilde{A})\right],
$$

where

$$
L^{N-1}(\tilde{A}) \equiv \mathbf{1}_{\left\{\alpha \tilde{A} \geq F^{N-1}(\tilde{A})\right\}} \alpha \tilde{A}+\mathbf{1}_{\left\{\alpha \tilde{A}<F^{N-1}(\tilde{A})\right\}} H^{N-1}(\tilde{A})
$$

We show the claim by induction. For $N=1$, (30)-(31) become

$$
\begin{aligned}
\left(T_{1}^{\bar{A}} g\right)(A) & =e^{-r \Delta} E_{\tilde{A} \mid A}\left[\mathbf{1}_{\{\tilde{A} \geq \bar{A}\}} g(\tilde{A})+\mathbf{1}_{\{\tilde{A}<\bar{A}\}} L^{0}(\tilde{A})\right], \\
L^{0}(\tilde{A}) & =\mathbf{1}_{\left\{\alpha \tilde{A} \geq F^{0}(\tilde{A})\right\}} \alpha \tilde{A}+\mathbf{1}_{\left\{\alpha \tilde{A}<F^{0}(\tilde{A})\right\}} H^{0}(\tilde{A}) .
\end{aligned}
$$

For monotonicity suppose $h(A) \leq g(A), \forall A>0$. Then

$$
\begin{aligned}
\left(T_{1}^{\bar{A}} h\right)(A) & =e^{-r \Delta} E_{\tilde{A} \mid A}\left[\mathbf{1}_{\{\tilde{A} \geq \bar{A}\}} h(\tilde{A})+\mathbf{1}_{\{\tilde{A}<\bar{A}\}} L^{0}(\tilde{A})\right] \\
& \leq e^{-r \Delta} E_{\tilde{A} \mid A}\left[\mathbf{1}_{\{\tilde{A} \geq \bar{A}\}} g(\tilde{A})+\mathbf{1}_{\{\tilde{A}<\bar{A}\}} L^{0}(\tilde{A})\right]=\left(T_{1}^{\bar{A}} g\right)(A) .
\end{aligned}
$$

For discounting, take any $g \in V$ and $a>0$. Then

$$
\begin{aligned}
\left(T_{1}^{\bar{A}}(g+a)\right)(A)= & e^{-r \Delta} E_{\tilde{A} \mid A}\left[\mathbf{1}_{\{\tilde{A} \geq \bar{A}\}}(g(\tilde{A})+a)+\mathbf{1}_{\{\tilde{A}<\bar{A}\}} L^{0}(\tilde{A})\right] \\
= & e^{-r \Delta} E_{\tilde{A} \mid A}\left[\mathbf{1}_{\{\tilde{A} \geq \bar{A}\}} g(\tilde{A})+\mathbf{1}_{\{\tilde{A}<\bar{A}\}} L^{0}(\tilde{A})\right] \\
& +e^{-r \Delta} a E_{\tilde{A} \mid A}\left[\mathbf{1}_{\{\tilde{A} \geq \bar{A}\}}\right]=\left(T_{1}^{\bar{A}} g\right)(A)+\beta a
\end{aligned}
$$


where $\beta \equiv e^{-r \Delta} E_{\tilde{A} \mid A}\left[\mathbf{1}_{\{\tilde{A} \geq \bar{A}\}}\right]<1$. Blackwell's condition of discounting holds because $\beta$ is strictly smaller than one. By Blackwell's theorem, $T_{1}^{\bar{A}}: V \rightarrow V$ is a contraction. Denote its unique fixed point as $h^{1}(\cdot ; \bar{A}) \in V$. Define sets

$$
\begin{aligned}
& W_{1} \equiv\left\{g \in V: \text { nondecreasing and } H^{0}(A) \leq g(A) \leq A \text { for all } A>0\right\} ; \text { and } \\
& W_{1}^{\prime} \equiv\left\{g \in V: \text { strictly increasing and } H^{0}(A)<g(A)<A \text { for all } A>0\right\} .
\end{aligned}
$$

Take $\bar{A} \leq \underline{A}^{1}$. By the same corollary as we used in Proposition 2.1, if we show $T_{1}^{\bar{A}}\left(W_{1}\right) \subset W_{1}^{\prime}$ then we have $h^{1}(\cdot ; \bar{A}) \in W_{1}^{\prime}$. So consider $T_{1}^{\bar{A}} g \in T_{1}^{\bar{A}}\left(W_{1}\right)$ for an arbitrary $g \in W_{1}$. By the assumption $\bar{A} \leq \underline{A}^{1}$, (32)-(33) reduce to

$$
\left(T_{1}^{\bar{A}} g\right)(A)=e^{-r \Delta} E_{\tilde{A} \mid A}\left[\mathbf{1}_{\{\tilde{A} \geq \bar{A}\}} g(\tilde{A})+\mathbf{1}_{\{\tilde{A}<\bar{A}\}} H^{0}(\tilde{A})\right] .
$$

Since $H^{0}$ is strictly increasing and $g \in W_{1}$, the expression inside the above expectation operator is increasing in $\tilde{A}$ and hence $T_{1}^{\bar{A}} g$ is strictly increasing. As was shown before, we have $\forall A>0, H^{0}(A)<A$. Also, $g \in W_{1}$ implies $\forall A>0, g(A) \leq A$. This implies $\forall A>0,\left(T_{1}^{\bar{A}} g\right)(A)<A$. Furthermore, we have

$$
\begin{aligned}
\left(T_{1}^{\bar{A}} g\right)(A) & \geq e^{-r \Delta} E_{\tilde{A} \mid A}\left[H^{0}(\tilde{A})\right] \\
& >e^{-r \Delta} E_{\tilde{A} \mid A}\left[\mathbf{1}_{\left\{\tilde{A} \geq \bar{A}^{0}\right\}} H^{0}(\tilde{A})+\mathbf{1}_{\left\{\tilde{A}<\bar{A}^{0}\right\}} \alpha \tilde{A}\right]=H^{0}(A) .
\end{aligned}
$$

Therefore we have $T_{1}^{\bar{A}} g \in W_{1}^{\prime}$, and hence $T_{1}^{\bar{A}}\left(W_{1}\right) \subset W_{1}^{\prime}$, and thus $h^{1}(\cdot ; \bar{A}) \in W_{1}^{\prime}$. That is, $h^{1}(\cdot ; \bar{A})$ is strictly increasing and satisfies $\forall A>0, H^{0}(A)<h^{1}(A ; \bar{A})<A$. In particular, $H^{0}(\bar{A})<h^{1}(\bar{A} ; \bar{A})<\bar{A}$. A version of the intermediate value theorem implies that there exists $A^{*}$ such that $h^{1}\left(A^{*} ; A^{*}\right)=f$, and $A^{*}$ satisfies $f<A^{*}<\bar{A}^{0}$. So let $\bar{A}^{1} \equiv A^{*}$ and $H^{1}(\cdot) \equiv h^{1}\left(\cdot ; \bar{A}^{1}\right)$. Under our assumption $\bar{A}^{0}<\underline{A}^{1}$, it follows that $\bar{A}^{1}<\underline{A}^{1}$. That is, if $\tilde{A}<\bar{A}^{1}$ so that the stockholder with $N=1$ chooses default, it is always the case that $\tilde{A}<\underline{A}^{1}$ so the creditor chooses to extend the maturity. ${ }^{18}$

Next we show $H^{1}(\cdot)>H^{\overline{0}}(\cdot)$. First, note that $H^{1}$ and $H^{0}$ are fixed points of the following two contractions, respectively:

$$
\begin{aligned}
& \left(T_{1}^{\bar{A}^{1}} g\right)(A)=e^{-r \Delta} E_{\tilde{A} \mid A}\left[\mathbf{1}_{\left\{\tilde{A} \geq \bar{A}^{1}\right\}} g(\tilde{A})+\mathbf{1}_{\left\{\tilde{A}<\bar{A}^{1}\right\}} H^{0}(\tilde{A})\right] \\
& \left(T_{0}^{\bar{A}^{0}} g\right)(A)=e^{-r \Delta} E_{\tilde{A} \mid A}\left[\mathbf{1}_{\left\{\tilde{A} \geq \bar{A}^{0}\right\}} g(\tilde{A})+\mathbf{1}_{\left\{\tilde{A}<\bar{A}^{0}\right\}} \alpha \tilde{A}\right] .
\end{aligned}
$$

Because $H^{0} \in W^{\prime}$, we have $\alpha \tilde{A}<H^{0}(\tilde{A})$ for all $\tilde{A}$. Therefore, $\bar{A}^{1}<\bar{A}^{0}$ implies that $\forall g \in V, \forall A>0,\left(T_{0}^{\bar{A}^{0}} g\right)(A)<\left(T_{1}^{\bar{A}^{1}} g\right)(A)$. Hence, applying Lemma 4.2 to $\Theta=\{0,1\}$ proves $H^{1}(\cdot)>H^{0}(\cdot)$.

\footnotetext{
18 Our computation shows that $\bar{A}^{0}<\underline{A}^{1}$ is true for small $\alpha$.
} 
Using this, we show $F^{1}(\cdot)>F^{0}(\cdot)$. This is true because

$$
\begin{aligned}
F^{1}(A) & =e^{-r \Delta} E_{\tilde{A} \mid A}\left[\mathbf{1}_{\left\{\tilde{A} \geq \bar{A}^{1}\right\}} f+\mathbf{1}_{\left\{\tilde{A}<\bar{A}^{1}\right\}} \max \left(\alpha \tilde{A}, F^{0}(\tilde{A})\right)\right] \\
& \geq e^{-r \Delta} E_{\tilde{A} \mid A}\left[\mathbf{1}_{\left\{\tilde{A} \geq \bar{A}^{1}\right\}} f+\mathbf{1}_{\left\{\tilde{A}<\bar{A}^{1}\right\}} \alpha \tilde{A}\right] \\
& >e^{-r \Delta} E_{\tilde{A} \mid A}\left[\mathbf{1}_{\left\{\tilde{A} \geq \bar{A}^{0}\right\}} f+\mathbf{1}_{\left\{\tilde{A}<\bar{A}^{0}\right\}} \alpha \tilde{A}\right]=F^{0}(A),
\end{aligned}
$$

where the second inequality follows from $f<\bar{A}^{1}<\bar{A}^{0}<f / \alpha$. This in turn implies $F^{1}(A)\left(>F^{0}(A)\right)>\alpha A$, for sufficiently small $A>0$ (Proposition 2.2). Now, note that

$$
\begin{aligned}
& S^{0}(A)=e^{-r \Delta} E_{\tilde{A} \mid A}\left[\mathbf{1}_{\left\{\tilde{A} \geq \bar{A}^{0}\right\}}\left(H^{0}(A)-f\right)\right] \\
& S^{1}(A)=e^{-r \Delta} E_{\tilde{A} \mid A}\left[\mathbf{1}_{\left\{\tilde{A} \geq \bar{A}^{1}\right\}}\left(H^{1}(A)-f\right)+\mathbf{1}_{\left\{\tilde{A}<\bar{A}^{1}\right\}} S^{0}(\tilde{A})\right] .
\end{aligned}
$$

Since $\bar{A}^{0}>\bar{A}^{1}$ and $H^{1}>H^{0}$, we have $S^{1}>S^{0}$. Let $A^{2} \equiv \min \{\tilde{A}>0$ : $\left.F^{1}(\tilde{A})-\alpha \tilde{A}=0\right\}$. Proposition 2.2 and $F^{1}(\cdot)>F^{0}(\cdot)$ imply $\underline{A}^{1}<\underline{A}^{2}$ and that $F^{1}(\tilde{A})>\alpha \tilde{A}$ for $\tilde{A} \in\left(0, \underline{A}^{2}\right)$. Therefore, once we show $\bar{A}^{2}<\bar{A}^{1}\left(<\bar{A}^{0}<\underline{A}^{1}<\underline{A}^{2}\right)$, it will follow that whenever the stockholder with $N=2$ chooses defaulting, the creditor chooses to extend maturity.

Next we turn to $N=2$ case and more general $N$ by induction. For $N=2$, (30)-(31) become

$$
\begin{gathered}
\left(T_{2}^{\bar{A}} g\right)(A)=e^{-r \Delta} E_{\tilde{A} \mid A}\left[\mathbf{1}_{\{\tilde{A} \geq \bar{A}\}} g(\tilde{A})+\mathbf{1}_{\{\tilde{A}<\bar{A}\}} L^{1}(\tilde{A})\right] \\
L^{1}(\tilde{A}) \equiv \mathbf{1}_{\left\{\alpha \tilde{A} \geq F^{1}(\tilde{A})\right\}} \alpha \tilde{A}+\mathbf{1}_{\left\{\alpha \tilde{A}<F^{1}(\tilde{A})\right\}} H^{1}(\tilde{A}) .
\end{gathered}
$$

Fix the threshold so that $\bar{A} \leq \bar{A}^{1}$. Then $\bar{A} \leq \bar{A}^{1} \leq \underline{A}^{1}<\underline{A}^{2}$. In this case, $\tilde{A}<\bar{A} \Rightarrow$ $\tilde{A}<\underline{A}^{2} \Rightarrow F^{1}(\tilde{A})>\alpha \tilde{A}$ holds, and hence (38)-(39) reduce to

$$
\left(T_{2}^{\bar{A}} g\right)(A)=e^{-r \Delta} E_{\tilde{A} \mid A}\left[\mathbf{1}_{\{\tilde{A} \geq \bar{A}\}} g(\tilde{A})+\mathbf{1}_{\{\tilde{A}<\bar{A}\}} H^{1}(\tilde{A})\right] .
$$

Define sets

$$
\begin{aligned}
& W_{2} \equiv\left\{g \in V: \text { nondecreasing and } H^{1}(A) \leq g(A) \leq A \text { for all } A>0\right\} ; \text { and } \\
& W_{2}^{\prime} \equiv\left\{g \in V: \text { strictly increasing and } H^{1}(A)<g(A)<A \text { for all } A>0\right\} .
\end{aligned}
$$

By similar arguments, it can be shown that $T_{2}^{\bar{A}}\left(W_{2}\right) \subset W_{2}^{\prime}$. So the fixed point is $h^{2}(\cdot ; \bar{A}) \in W_{2}^{\prime}$. Hence $H^{1}(A)<h^{2}(A ; \bar{A})<A$ for all $A>0$, or in particular, $H^{1}(\bar{A})<h^{2}(\bar{A} ; \bar{A})<\bar{A}$. This implies that there exists $\bar{A}^{2}$ such that $h^{2}\left(\bar{A}^{2} ; \bar{A}^{2}\right)=f$ and $f<\bar{A}^{2}<\bar{A}^{1}$. Because $\bar{A}^{2}<\underline{A}^{2}$, the creditor always chooses to extend maturity. Let $H^{2}(\cdot) \equiv h^{2}\left(\cdot ; \bar{A}^{2}\right)$ and show $\overline{H^{2}}(\cdot)>H^{1}(\cdot)$ and $F^{2}(\cdot)>F^{1}(\cdot)$, and so on. 
Proof of Proposition 2.4 We look for a solution in which the creditor always chooses to exercise the maturity extension option whenever $M \geq 1$. So assume that in (14)(15) $\alpha \tilde{A}<F^{N, M-1}(\tilde{A})$ always holds whenever $\tilde{A}<\bar{A}^{R, N}$, which should be verified later. Also, let $H^{N, M}(A) \equiv F^{N, M}(A)+S^{N, M}(A)$. Equations (14)-(15) then reduce to

$$
\begin{aligned}
& F^{N, M}(A)= \begin{cases}e^{-r \Delta} E_{\tilde{A} \mid A}\left[\mathbf{1}_{\left\{\tilde{A} \geq \bar{A}^{R, N\}}\right.} f+\mathbf{1}_{\left\{\tilde{A}<\bar{A}^{R, N}\right\}} \alpha \tilde{A}\right] & \text { if } M=0 \\
e^{-r \Delta} E_{\tilde{A} \mid A}\left[\mathbf{1}_{\left\{\tilde{A} \geq \bar{A}^{R, N}\right\}} f+\mathbf{1}_{\left\{\tilde{A}<\bar{A}^{R, N}\right\}} F^{N, M-1}(\tilde{A})\right] & \text { if } M \geq 1\end{cases}
\end{aligned}
$$

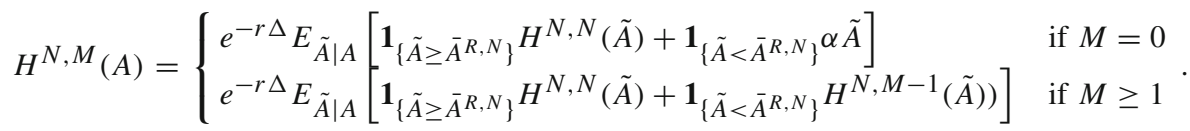

For a given $N,(41)$ implies that $N+1$ equations, $H^{N, 0}, \ldots, H^{N, N}$, are dependent on one another. Therefore, we must construct a contraction mapping that is applied to a vector of $N+1$ functions. Define a mapping $P^{\bar{A}}: V^{1+N} \rightarrow V^{1+N}$ by the r.h.s. of (41) with $\bar{A}^{R, N}$ replaced by an arbitrary threshold $\bar{A}$ that is no greater than $\bar{A}^{0}$ :

$$
\begin{aligned}
& P_{0}^{\bar{A}}\left(g^{0}, \ldots, g^{N}\right)(A)=e^{-r \Delta} E_{\tilde{A} \mid A}\left[\mathbf{1}_{\{\tilde{A} \geq \bar{A}\}} g^{N}(\tilde{A})+\mathbf{1}_{\{\tilde{A}<\bar{A}\}} \alpha \tilde{A}\right], \quad M=0 \\
& \left.P_{M}^{\bar{A}}\left(g^{0}, \ldots, g^{N}\right)(A)=e^{-r \Delta} E_{\tilde{A} \mid A}\left[\mathbf{1}_{\{\tilde{A} \geq \bar{A}\}} g^{N}(\tilde{A})+\mathbf{1}_{\{\tilde{A}<\bar{A}\}} g^{M-1}(\tilde{A})\right)\right], \quad M \geq 1 .
\end{aligned}
$$

$P^{\bar{A}}$ satisfies monotonicity and discounting. Monotonicity is straightforward. For discounting, note that for $M=0$ and $a \in \mathbb{R}_{++}^{1+N}$,

$$
\begin{aligned}
& P_{0}^{\bar{A}}\left(g^{0}+a_{1}, \ldots, g^{N}+a_{N}\right)(A) \\
& =e^{-r \Delta} E_{\tilde{A} \mid A}\left[\mathbf{1}_{\{\tilde{A} \geq \bar{A}\}}\left(g^{N}(\tilde{A})+a_{N}\right)+\mathbf{1}_{\{\tilde{A}<\bar{A}\}} \alpha \tilde{A}\right] \\
& =e^{-r \Delta} E_{\tilde{A} \mid A}\left[\mathbf{1}_{\{\tilde{A} \geq \bar{A}\}} g^{N}(\tilde{A})+\mathbf{1}_{\{\tilde{A}<\bar{A}\}} \alpha \tilde{A}\right]+e^{-r \Delta} a_{N} E_{\tilde{A} \mid A}\left[\mathbf{1}_{\{\tilde{A} \geq \bar{A}\}}\right] \\
& \leq P^{\bar{A}}\left(g^{0}, \ldots, g^{N}\right)(A)+\beta \max _{j} a_{j},
\end{aligned}
$$

where $\beta=e^{-r \Delta} E_{\tilde{A} \mid A}\left[\mathbf{1}_{\{\tilde{A} \geq \bar{A}\}}\right]<1$. For $M \geq 1$,

$$
\begin{aligned}
P_{M}^{\bar{A}} & \left(g^{0}+a_{1}, \ldots, g^{N}+a_{N}\right)(A) \\
= & e^{-r \Delta} E_{\tilde{A} \mid A}\left[\mathbf{1}_{\{\tilde{A} \geq \bar{A}\}}\left(g^{N}(\tilde{A})+a_{N}\right)+\mathbf{1}_{\{\tilde{A}<\bar{A}\}}\left(g^{M-1}(\tilde{A})+a_{M-1}\right)\right] \\
= & e^{-r \Delta} E_{\tilde{A} \mid A}\left[\mathbf{1}_{\{\tilde{A} \geq \bar{A}\}} g^{N}(\tilde{A})\right. \\
& \left.+\mathbf{1}_{\{\tilde{A}<\bar{A}\}} g^{M-1}(\tilde{A})\right]+e^{-r \Delta}\left(a_{N} E_{\tilde{A} \mid A}\left[\mathbf{1}_{\{\tilde{A} \geq \bar{A}\}}\right]+a_{M-1} E_{\tilde{A} \mid A}\left[\mathbf{1}_{\{\tilde{A}<\bar{A}\}}\right]\right) \\
\leq & P_{M}^{\bar{A}}\left(g^{0}, \ldots, g^{N}\right)(A)+\beta \max _{j} a_{j},
\end{aligned}
$$


where $\beta=e^{-r \Delta}<1$. By Lemma 4.1, $P^{\bar{A}}$ is a contraction. Define sets $Z_{1}^{\prime} \subset Z_{1} \subset$ $V^{1+N}$ by

$$
\begin{aligned}
Z_{1} & \equiv\left\{\left(g^{0}, \ldots, g^{N}\right): H^{0}(A) \leq g^{0}(A)\right. \\
& \left.\leq \cdots \leq g^{N}(A) \leq A, \forall A, \text { and } g^{\prime} \text { s are nondecreasing. }\right\}
\end{aligned}
$$

and

$$
\begin{aligned}
Z_{1}^{\prime} & \equiv\left\{\left(g^{0}, \ldots, g^{N}\right): H^{0}(A)<g^{0}(A)<\cdots<g^{N}(A)\right. \\
& \left.<A, \forall A, \text { and } g^{\prime} \text { s are strictly increasing. }\right\} .
\end{aligned}
$$

Then since $\bar{A} \leq \bar{A}^{0}$, for any $G=\left(g^{0}, \ldots, g^{N}\right) \in Z_{1}$, we have

$$
\begin{aligned}
P_{0}^{\bar{A}}\left(g^{0}, \ldots, g^{N}\right)(A) & =e^{-r \Delta} E_{\tilde{A} \mid A}\left[\mathbf{1}_{\{\tilde{A} \geq \bar{A}\}} g^{N}(\tilde{A})+\mathbf{1}_{\{\tilde{A}<\bar{A}\}} \alpha \tilde{A}\right] \\
& \geq e^{-r \Delta} E_{\tilde{A} \mid A}\left[\mathbf{1}_{\left\{\tilde{A} \geq \bar{A}^{0}\right\}} H^{0}(\tilde{A})+\mathbf{1}_{\left\{\tilde{A}<\bar{A}^{0}\right\}} \alpha \tilde{A}\right]=H^{0}(A),
\end{aligned}
$$

[see Eq. (22)], and

$$
\begin{aligned}
P_{0}^{\bar{A}}\left(g^{0}, \ldots, g^{N}\right)(A) & =e^{-r \Delta} E_{\tilde{A} \mid A}\left[\mathbf{1}_{\{\tilde{A} \geq \bar{A}\}} g^{N}(\tilde{A})+\mathbf{1}_{\{\tilde{A}<\bar{A}\}} \alpha \tilde{A}\right] \\
& <e^{-r \Delta} E_{\tilde{A} \mid A}[\tilde{A}]=A .
\end{aligned}
$$

Next, we have

$$
\begin{aligned}
P_{1}^{\bar{A}}\left(g^{0}, \ldots, g^{N}\right)(A) & =e^{-r \Delta} E_{\tilde{A} \mid A}\left[\mathbf{1}_{\{\tilde{A} \geq \bar{A}\}} g^{N}(\tilde{A})+\mathbf{1}_{\{\tilde{A}<\bar{A}\}} g^{0}(\tilde{A})\right] \\
& >e^{-r \Delta} E_{\tilde{A} \mid A}\left[\mathbf{1}_{\{\tilde{A} \geq \bar{A}\}} g^{N}(\tilde{A})+\mathbf{1}_{\{\tilde{A}<\bar{A}\}} \alpha \tilde{A}\right] \\
& =P_{0}^{\bar{A}}\left(g^{0}, \ldots, g^{N}\right)(A),
\end{aligned}
$$

and

$$
\begin{aligned}
P_{1}^{\bar{A}}\left(g^{0}, \ldots, g^{N}\right)(A) & =e^{-r \Delta} E_{\tilde{A} \mid A}\left[\mathbf{1}_{\{\tilde{A} \geq \bar{A}\}} g^{N}(\tilde{A})+\mathbf{1}_{\{\tilde{A}<\bar{A}\}} g^{0}(\tilde{A})\right] \\
& \leq e^{-r \Delta} E_{\tilde{A} \mid A}[\tilde{A}]=A,
\end{aligned}
$$

where the inequality is strict if $g^{0}<A$. Furthermore, for $M \geq 2$,

$$
\begin{aligned}
P_{M}^{\bar{A}}\left(g^{0}, \ldots, g^{N}\right)(A) & =e^{-r \Delta} E_{\tilde{A} \mid A}\left[\mathbf{1}_{\{\tilde{A} \geq \bar{A}\}} g^{N}(\tilde{A})+\mathbf{1}_{\{\tilde{A}<\bar{A}\}} g^{M-1}(\tilde{A})\right] \\
& \geq e^{-r \Delta} E_{\tilde{A} \mid A}\left[\mathbf{1}_{\{\tilde{A} \geq \bar{A}\}} g^{N}(\tilde{A})+\mathbf{1}_{\{\tilde{A}<\bar{A}\}} g^{M-2}(\tilde{A})\right] \\
& =P_{M-1}^{\bar{A}}\left(g^{0}, \ldots, g^{N}\right)(A),
\end{aligned}
$$


where the inequality is strict if $g^{M-2}<g^{M-1}$, and

$$
\begin{aligned}
P_{M}^{\bar{A}}\left(g^{0}, \ldots, g^{N}\right)(A) & =e^{-r \Delta} E_{\tilde{A} \mid A}\left[\mathbf{1}_{\{\tilde{A} \geq \bar{A}\}} g^{N}(\tilde{A})+\mathbf{1}_{\{\tilde{A}<\bar{A}\}} g^{M-1}(\tilde{A})\right] \\
& \leq e^{-r \Delta} E_{\tilde{A} \mid A}[\tilde{A}]=A,
\end{aligned}
$$

where the inequality is strict if $g^{M-1}<A$. Lastly, we have

$$
\begin{aligned}
P_{0}^{\bar{A}}\left(g^{0}, \ldots, g^{N}\right)(A) & =e^{-r \Delta} E_{\tilde{A} \mid A}\left[\mathbf{1}_{\{\tilde{A} \geq \bar{A}\}} g^{N}(\tilde{A})+\mathbf{1}_{\{\tilde{A}<\bar{A}\}} \alpha \tilde{A}\right] \\
& <e^{-r \Delta} E_{\tilde{A} \mid A^{\prime}}\left[\mathbf{1}_{\{\tilde{A} \geq \bar{A}\}} g^{N}(\tilde{A})+\mathbf{1}_{\{\tilde{A}<\bar{A}\}} \alpha \tilde{A}\right] \\
& =P_{0}^{\bar{A}}\left(g^{0}, \ldots, g^{N}\right)\left(A^{\prime}\right)
\end{aligned}
$$

if $A<A^{\prime}$, and

$$
\begin{aligned}
P_{M}^{\bar{A}}\left(g^{0}, \ldots, g^{N}\right)(A) & =e^{-r \Delta} E_{\tilde{A} \mid A}\left[\mathbf{1}_{\{\tilde{A} \geq \bar{A}\}} g^{N}(\tilde{A})+\mathbf{1}_{\{\tilde{A}<\bar{A}\}} g^{M-1}(\tilde{A})\right] \\
& <e^{-r \Delta} E_{\tilde{A} \mid A^{\prime}}\left[\mathbf{1}_{\{\tilde{A} \geq \bar{A}\}} g^{N}(\tilde{A})+\mathbf{1}_{\{\tilde{A}<\bar{A}\}} g^{M-1}(\tilde{A})\right] \\
& =P_{M}^{\bar{A}}\left(g^{0}, \ldots, g^{N}\right)\left(A^{\prime}\right)
\end{aligned}
$$

so the result of the mapping is strictly increasing. All the above together imply that applying $P^{\bar{A}}$ on $Z_{1}$ repeatedly $N$ times guarantees $\left(P^{\bar{A}}\right)^{N}\left(Z_{1}\right) \subset Z_{1}^{\prime}$. Therefore, the fixed point of $P^{\bar{A}}$, say $\left(h^{N, 0}(\cdot ; \bar{A}), \ldots, h^{N, N}(\cdot ; \bar{A})\right)$, is in $Z_{1}^{\prime}$. That is, for all $A>0$,

$$
H^{0}(A)<h^{N, 0}(A ; \bar{A})<\cdots<h^{N, N}(A ; \bar{A})<A
$$

and in particular

$$
H^{0}(\bar{A})<h^{N, 0}(\bar{A} ; \bar{A})<\cdots<h^{N, N}(\bar{A} ; \bar{A})<\bar{A} .
$$

Letting $\bar{A}^{R, N}$ be such that $h^{N, N}\left(\bar{A}^{R, N} ; \bar{A}^{R, N}\right)=f$, we have

$$
\left(\alpha \bar{A}^{R, N}<\alpha \bar{A}^{0}<\right) f<\bar{A}^{R, N}<\bar{A}^{0} .
$$

Also, (44) holds for this particular threshold and we have $H^{0}(A)<h^{N, 0}\left(A ; \bar{A}^{R, N}\right)$ $<\cdots<h^{N, N}\left(A ; \bar{A}^{R, N}\right)<A$, or

$$
H^{0}(A)<H^{N, 0}(A)<\cdots<H^{N, N}(A)<A .
$$

Now we turn to debt values. We have

$$
\begin{aligned}
F^{N, 0}(A) & =e^{-r \Delta} E_{\tilde{A} \mid A}\left[\mathbf{1}_{\left\{\tilde{A} \geq \bar{A}^{R, N}\right\}} f+\mathbf{1}_{\left\{\tilde{A}<\bar{A}^{R, N}\right\}} \alpha \tilde{A}\right] \\
& >e^{-r \Delta} E_{\tilde{A} \mid A}\left[\mathbf{1}_{\left\{\tilde{A} \geq \bar{A}^{0}\right\}} f+\mathbf{1}_{\left\{\tilde{A}<\bar{A}^{0}\right\}} \alpha \tilde{A}\right]=F^{0}(A)
\end{aligned}
$$


where the inequality comes from $\bar{A}^{R, N}<\bar{A}^{0}<f / \alpha$. Also

$$
\begin{aligned}
F^{N, 1}(A) & =e^{-r \Delta} E_{\tilde{A} \mid A}\left[\mathbf{1}_{\left\{\tilde{A} \geq \bar{A}^{R, N}\right\}} f+\mathbf{1}_{\left\{\tilde{A}<\bar{A}^{R, N}\right\}} F^{N, 0}(\tilde{A})\right] \\
& >e^{-r \Delta} E_{\tilde{A} \mid A}\left[\mathbf{1}_{\left\{\tilde{A} \geq \bar{A}^{R, N}\right\}} f+\mathbf{1}_{\left\{\tilde{A}<\bar{A}^{R, N}\right\}} \alpha \tilde{A}\right]=F^{N, 0}(A),
\end{aligned}
$$

where the inequality comes from $\left(\tilde{A}<\bar{A}^{R, N}<\bar{A}^{0}<\underline{A}^{1}\right) \Rightarrow\left(F^{N, 0}(\tilde{A})>F^{0}(\tilde{A})>\right.$ $\alpha \tilde{A})$. By induction, we have $F^{N, M}(\cdot)>F^{N, M-1}(\cdot), M=1, \ldots, N$. By letting $\underline{A}^{N, M} \equiv \min \left\{\tilde{A}>0: F^{N, M}(\tilde{A})-\alpha \tilde{A}=0\right\}$, we have $\bar{A}^{R, N}<\bar{A}^{0}<\underline{A}^{N, 1}<$ $\bar{A}^{N, 2}<\cdots$. Therefore the initial assumption that the creditor always chooses to extend the maturity has been confirmed. Since $S^{N, M}(\cdot)=H^{N, M}(\cdot)-F^{N, M}(\cdot)$, we have

$$
S^{N, 0}(A)=e^{-r \Delta} E_{\tilde{A} \mid A}\left[\mathbf{1}_{\left\{\tilde{A} \geq \bar{A}^{R, N}\right\}}\left(H^{N, N}(\tilde{A})-f\right)\right],
$$

so $S^{N, 0}$ is nonnegative-valued and strictly increasing. Also

$$
\begin{aligned}
S^{N, 1}(A) & =e^{-r \Delta} E_{\tilde{A} \mid A}\left[\mathbf{1}_{\{\tilde{A} \geq \bar{A} R, N\}}\left(H^{N, N}(\tilde{A})-f\right)+\mathbf{1}_{\{\tilde{A}<\bar{A} R, N\}} S^{N, 0}(\tilde{A})\right] \\
& >S^{N, 0}(A) .
\end{aligned}
$$

where the inequality follows (52). Then by induction, we have $S^{N, M}>S^{N, M-1}$ for $M=1, \ldots, N$.

Proof of Proposition 2.5 We impose $\bar{A}^{\infty}=f$ and verify it later. Let $V$ be the set of functions defined on $\mathbb{R}_{++}$that are positive-valued and bounded by $f$. Define a mapping $T_{\infty}: V \rightarrow V$ by

$$
\left(T_{\infty} g\right)(A) \equiv e^{-r \Delta} E_{\tilde{A} \mid A}\left[\mathbf{1}_{\{\tilde{A} \geq f\}} f+\mathbf{1}_{\{\tilde{A}<f\}} \max (\alpha \tilde{A}, g(\tilde{A}))\right] .
$$

The monotonicity of $T_{\infty}$ is obvious and the discounting follows from

$$
\begin{aligned}
\left(T_{\infty}(g+a)\right)(A) & =e^{-r \Delta} E_{\tilde{A} \mid A}\left[\mathbf{1}_{\{\tilde{A} \geq f\}} f+\mathbf{1}_{\{\tilde{A}<f\}} \max (\alpha \tilde{A}, g(\tilde{A})+a)\right] \\
& \leq e^{-r \Delta} E_{\tilde{A} \mid A}\left[\mathbf{1}_{\{\tilde{A} \geq f\}} f+\mathbf{1}_{\{\tilde{A}<f\}} \max (\alpha \tilde{A}+a, g(\tilde{A})+a)\right] \\
& =e^{-r \Delta} E_{\tilde{A} \mid A}\left[\mathbf{1}_{\{\tilde{A} \geq f\}} f+\mathbf{1}_{\{\tilde{A}<f\}}(\max (\alpha \tilde{A}, g(\tilde{A}))+a)\right] \\
& =\left(T_{\infty} g\right)(A)+\beta a,
\end{aligned}
$$

where $\beta=e^{-r \Delta} E_{\tilde{A} \mid A}\left[\mathbf{1}_{\{\tilde{A}<f\}}\right]<1$. Therefore, by the Blackwell sufficient condition, a unique fixed point exists, denoted $F^{\infty}(\cdot)$. Moreover, for all $A>0$, we have

$$
\begin{aligned}
F^{\infty}(A) & >e^{-r \Delta} E_{\tilde{A} \mid A}\left[\mathbf{1}_{\{\tilde{A} \geq f\}} f+\mathbf{1}_{\{\tilde{A}<f\}} \alpha \tilde{A}\right] \\
& >e^{-r \Delta} E_{\tilde{A} \mid A}\left[\mathbf{1}_{\left\{\tilde{A} \geq \bar{A}^{0}\right\}} f+\mathbf{1}_{\left\{\tilde{A}<\bar{A}^{0}\right\}} \alpha \tilde{A}\right]=F^{0}(A) .
\end{aligned}
$$


Hence $F^{\infty}>F^{0}$. That is, if we define $\underline{A}^{\infty} \equiv \inf \left\{\tilde{A}>0: F^{\infty}(\tilde{A})<\alpha \tilde{A}\right\}$, then we have $\bar{A}^{\infty}=f<\bar{A}^{0}<\underline{A}^{1}<\underline{A}^{\infty}$. That is, $\tilde{A}<\bar{A}^{\infty} \Rightarrow \alpha \tilde{A}<F^{\infty}(\tilde{A})$; whenever default occurs, the creditor chooses postponement. In this case, summing up (19) and (21) gives

$$
F^{\infty}(A)+S^{\infty}(A)=e^{-r \Delta} E_{\tilde{A} \mid A}\left[F^{\infty}(\tilde{A})+S^{\infty}(\tilde{A})\right] .
$$

Given that the expectation is with respect to the risk-neutral probability measure, the obvious solution is $F^{\infty}(A)+S^{\infty}(A)=A$ for all $A>0$. Then, it is verified that $\bar{A}^{\infty}=f$ satisfies the definition of the default threshold, namely $S^{\infty}\left(\bar{A}^{\infty}\right)-(f-$ $\left.F^{\infty}\left(\bar{A}^{\infty}\right)\right)=0$.

\section{References}

Aoki, M., Hugh, P.: The Japanese Main Bank System: Its Relevance for Developing and Transforming Economies. Oxford University Press, Oxford (1995)

Black, F., Cox, J.C.: Valuing corporate securities: some effects of bond indenture provisions. J Finance 31(2), 351-367 (1976)

Black, F., Scholes M.: The pricing of options and corporate liabilities. J Polit Econ 81(3), 637-654 (1973)

Brennan, M.J., Schwartz, E.S.: Corporate income taxes, valuation, and the problem of optimal capital structure. J Bus 51(1), 103-114 (1978)

Fan, H., Sundaresan, S.M.: Debt valuation, renegotiation, and optimal dividend policy. Rev Financ Stud 13(4), 1057-1099 (2000)

Kim, J., Ramaswamy, K., Sundaresan, S.: Does default risk in coupons affect the valuation of corporate bonds?: a contingent claims model. Financ Manag 22(3), 117-131 (1993)

Leland, H.: Corporate debt value, bond covenants and optimal capital structure. J Finance 49(4), 1213-1252 (1994)

Leland, H., Toft, K.: Optimal capital structure, endogenous bankruptcy and the term structure of credit spreads. J Finance 51(3), 987-1019 (1996)

Longstaff, F.A., Schwartz, E.S.: A simple approach to valuing risky fixed and floating rate debt. J Finance 50(3), 789-819 (1995)

Mella-Barral, P., Perraudin, W.: Strategic debt service. J Finance 52(2), 531-556 (1997)

Merton, R.: On the pricing of corporate debt: the risk structure of interest rates. J Finance 29(2), 449-470 (1974)

Stokey, N., Lucas, R., Prescott, E.: Recursive Methods in Economic Dynamics. Harvard University Press, Cambridge (1989)

Turnbull, S. M.: Debt capacity. J Finance 34(4), 931-940 (1979) 